\title{
Chaves de identificação de larvas para famílias e gêneros de Trichoptera (Insecta) da Amazônia Central, Brasil
}

\author{
Ana Maria Oliveira Pes ${ }^{1}$, Neusa Hamada² \& Jorge Luiz Nessimian ${ }^{3}$
}

\begin{abstract}
${ }^{1}$ Divisão de Curso em Entomologia, Instituto Nacional de Pesquisas da Amazônia, Caixa Postal 478, 69011-970 Manaus-AM, Brasil. pesanamaria@yahoo.com.br

${ }^{2}$ Coordenação de Pesquisas em Entomologia, Instituto Nacional de Pesquisas da Amazônia, Caixa Postal 478, 69011-970 Manaus-AM, Brasil. nhamada@inpa.gov.br

${ }^{3}$ Laboratório de Entomologia, Departamento de Zoologia, Instituto de Biologia, Universidade Federal do Rio de Janeiro, Caixa Postal 68044, 21944-970 Rio de Janeiro-RJ, Brasil. nessimia@acd.ufrj.br
\end{abstract}

\begin{abstract}
Identification key to families and genera of larvae of Trichoptera from Central Amazonia, Brazil. Keys for identification of larvae to families and genera of Trichoptera of Central Amazonia are presented.

KEYWORDS. Aquatic Insects; Central Amazonian; larvae; taxonomy; Trichoptera.

RESUMO. Chaves de identificação de larvas para famílias e gêneros de Trichoptera (Insecta) da Amazônia Central, Brasil. Chaves de identificação de larvas aos níveis de família e gênero de Trichoptera da Amazônia Central são apresentadas.
\end{abstract}

PALAVRAS-CHAVE. Amazônia Central; insetos aquáticos; larvas; taxonomia; Trichoptera.

Os Trichoptera surgiram no Triássico e compartilham um ancestral comum com Lepidoptera (Resh \& Solem 1996). Larvas apresentam cabeça esclerotinizada, antenas muito curtas, mandíbulas bem desenvolvidas, palpos maxilares reduzidos, tórax com o pronoto esclerotinizado, meso e metanoto com esclerotinização variável ou ausente. O abdome, membranoso, pode apresentar brânquias e o segmento IX tem um par de falsas pernas anais providas de garras (Angrisano 1998). Vivem em ambientes dulcícolas lóticos e lênticos, raras espécies são marinhas (Flint et al. 1999). Produzem seda que é usada na construção de casas, abrigos ou redes para retenção de partículas alimentares. Alguns grupos só constroem casa no último estádio larval (Angrisano 1998; Wiggins 1996).

Foram registradas 130 espécies de Trichoptera para a região norte, sendo 106 no estado do Amazonas e 53 em áreas próximas à Manaus (Flint et al. 1999; Prather 2003). Esses números indicam grande riqueza de espécies na região, pois estes trabalhos foram baseados em coletas esporádicas.

Trabalhos com descrições de Trichoptera da região amazônica foram realizados por Ulmer $(1905,1906)$ Martynov (1912), Sattler (1963a, b), Flint (1971, 1978, 1991), Sattler \& Sykora (1977). Outros trabalhos taxonômicos, com descrição de larvas e adultos foram realizados em países vizinhos como: Venezuela, Equador (Botosaneanu \& Flint 1982), Peru (Flint 1996; Flint \& Reyes 1991), Suriname (Flint 1974) e Colômbia (Flint 1981; Flint \& Bueno-soria 1982).

A maioria das espécies da região amazônica foi descrita no estágio adulto, os imaturos e sua biologia são quase que desconhecidos e as chaves de identificação disponíveis, principalmente, para larvas são da região neártica e da Argentina (Angrisano 1995; Wiggins 1996).

Este trabalho tem por objetivo fornecer chaves de identificação de famílias e gêneros de larvas de último estádio dos Trichoptera coletados em três municípios da Amazônia Central, contribuindo para o conhecimento taxonômico do grupo.

Como muitas larvas de Trichoptera são sensíveis a alterações ambientais em cursos d'água e esta resposta é especifica a identificação mesmo que genérica é imprescindível. Esta chave será um instrumento para auxiliar na identificação desses insetos em trabalhos de ecologia e avaliação de impacto ambiental.

\section{MATERIAL E MÉTODOS}

As larvas de Trichoptera foram coletadas em igarapés do município de Presidente Figueiredo (01 ${ }^{\circ} 59^{\prime} \mathrm{S} / 60^{\circ} 01^{\prime} \mathrm{W}$ ), localizado a $100 \mathrm{~km}$ ao norte de Manaus, no período de fevereiro 2000 a janeiro de 2002; em abril-maio de 2001 nos igarapés da Reserva Florestal Adolpho Ducke (02 53 'S $/ 59^{\circ} 58^{\prime} \mathrm{W}$ ) a $26 \mathrm{~km}$ de Manaus e setembro de 2001, nos igarapés do Projeto Dinâmica Biológica de Fragmentos Florestais (PDBFF), nos municípios de Manaus (Fazenda Dimona: $02^{\circ} 19^{\prime} \mathrm{S} / 60^{\circ} 04^{\prime} \mathrm{W}$ ) e Rio Preto da Eva (Reserva do km 41 - ZF2: $02^{\circ} 26^{\prime} \mathrm{S} / 59^{\circ} 46^{\prime} \mathrm{W}$ ).

As larvas foram examinadas sob estereomicroscópio e desenhadas com auxílio de câmara lúcida. Larvas pequenas foram dissecadas e algumas clarificadas para permitir observar estruturas como antenas, pernas e cerdas. Estas foram colocadas em lâminas escavadas com uma gota de glicerina para observação sob microscópio óptico e posteriormente montadas em lâminas permanentes utilizando Euparal ${ }^{\circledR}$, como meio de montagem.

Para a identificação das famílias e gêneros foram utilizados, principalmente, os trabalhos de Angrisano (1995), Flint (1963, 
1983), Flint \& Harris (1992), Flint et al. (1994), Flint \& Wallace (1980), Guahyba, (1991), Holzenthal (1995), Holzenthal \& Pes, (2004), Marlier (1964), Pes \& Hamada (2003, 2004), Roback (1966), Sattler (1963a, b) e Wiggins (1996). Quando necessário a identificação dos gêneros foi realizada por meio da associação das larvas com os adultos farados.

As larvas estão depositadas na Coleção de Invertebrados do Instituto Nacional de Pesquisas da Amazônia (INPA), Manaus, AM.

As figuras foram dispostas por ordem alfabética de famílias e gêneros. Gêneros citados como sp. 1, sp. 2, correspondem a larvas morfotipadas por PES (2001).

\section{RESULTADOS}

Foram identificados 13 famílias e 33 gêneros na área de estudo (em Leptoceridae e Sericostomatidae dois gêneros foram morfotipados, pois não foi possível a identificação).

Chave de identificação para larvas de último estádio de famílias e gêneros de Trichoptera da Amazônia Central (adaptada de Wiggins 1996).

1. Larva com abrigo móvel de areia em forma de caracol (Fig. 25); abdome encurvado (Fig. 23); garra da falsa perna anal em forma de pente (Fig. 24)

Helicopsyche Sielbold - Helicopsychidae

Larva com abrigo ausente ou de outra forma; abdome não encurvado; garra da falsa perna anal não como acima

2(1). Dorso do tórax recoberto com três placas inteiras esclerotinizadas (Figs. 5, 29, 31, 33, 36, 39, 41, 47, 48, $50,52,57,62,69,70,74,79,82,89,95,100)$

Somente o pronoto com placa inteira esclerotinizada, meta e mesonoto membranosos (Figs. 26, 28, 134, $135,138,147,151,152,162,163)$ ou com placas subdivididas (Figs. 2, 8, 13, 19, 104, 107, 110, 111, $115,119,125,130,159)$ 5

3(2). Abdome com brânquias ventro-laterais; garra da falsa perna anal com tufo de cerdas longas (Figs. 29, 33, $36,41,47)$. Hydropsychidae

Abdome sem brânquias (Figs. 5, 62, 74, 82, 89); garra da falsa perna anal sem tufo de cerdas longas ........ 4

4(3). Larvas com cerca de $15 \mathrm{~mm}$ de comprimento (Fig. 5); trocatim longo, mais longo que a coxa da perna anterior, em forma de canaleta, protegendo a coxa (Fig. 6) .............. Austrotinodes Schmid-Ecnomidae Larvas pequenas, no máximo com $3 \mathrm{~mm}$ de comprimento (Figs. 50-103); trocantim geralmente truncado ou pontiagudo, nunca como acima .......... Hydroptilidae

5(2). Mesonoto recoberto por placas esclerotinizadas recobrindo mais da metade do noto (Figs. 2, 107, 111, $115,119,125,130,159)$
Mesonoto sem placas esclerotinizadas (Figs. 28, 135, $138,147,152,163)$, ou com pequenos escleritos cobrindo não mais do que a metade do noto (Figs. 8, $13,19)$ ... 6

6(5). Segmento abdominal IX com uma placa esclerotinizada na região dorsal (Figs. 7, 14, 18, 27) .... 7

Segmento abdominal IX sem placa esclerotinizada na região dorsal (Figs. 141, 153) 8

7(6). Porção basal das falsas pernas anais livre; garra da falsa perna anal longa (Fig. 26); garra do primeiro par de pernas em forma de pinça (Fig. 26); larva de vida livre sem abrigo.... Atopsyche Banks - Hydrobiosidae

Porção basal das falsas pernas anais unidas ao segmento IX (Fig. 14); garra da falsa perna anal curta, unida ao abdome; abrigo de grãos de areia com duas aberturas ventrais, com formato semelhante a casco de tartaruga (Figs. 11, 17, 22) ......... Glossosomatidae

8(6). Labro membranoso em forma de T (Figs. 135, 139) ....... Philopotamidae

Labro membranoso arredondado 9

9(8). Tíbia e tarso fundidos em todas as pernas; mesopleura expandida anteriormente formando um processo lobado (Figs. 162, 163); palpo labial em forma de tubo afunilado (Figs. 163, 164); abrigo longo e estreito, constituído de seda, areia e sedimento (Fig.165) .....

....................................... Machairocentron

Schmid e Xiphocentron Brauer - Xiphocentronidae

Tíbia e tarso não fundidos; mesopleura não modificada como acima (Figs. 146, 151), palpo labial normal; abrigo, quando presente, de outra forma Polycentropodidae

10(5). Antenas longas, pelo menos 6 vezes a largura, (Figs. $105,110,115,119,125)$. Leptoceridae

Antenas curtas, não mais que 3 vezes a largura, ou não aparentes 11

11(10). Labro com fileira transversal central de aproximadamente 16 cerdas longas (Fig. 2); pronoto com uma projeção anterior (Fig. 2); abrigo de fragmentos de folhas sobrepostas (Figs. 3, 4) ...... Phylloicus Müller-Calamoceratidae

Labro com no máximo 8 cerdas (Figs. 130, 157), pronoto sem projeção como acima; abrigo de outra forma .. 12

12(11). Trocantim muito pequeno, pouco visível; regiões ventro-lateral e dorsal dos segmentos abdominais II-IX, com muitos filamentos branquiais ramificados na base (Fig. 129); abrigo de areia, recurvado e resistente (Fig. 133) . Marilia Müller-Odontoceridae

Trocantim visível, em forma de gancho, região ventrolateral dos segmentos abdominais I- IV com brânquias 
finas e de difícil visualização, não ramificados na base (Fig. 156); abrigo de areia, recurvado e frágil (Fig. 161) .............................. gênero A - Sericostomatidae

Chave de identificação para larvas de último estádio para os gêneros de Glossosomatidae

1. Garra da falsa perna anal composta por 1 processo e 2 pares de garras acessórias (Fig. 15), garras tarsais com cerda articulada longa e grossa (Fig. 16) Mortoniella Ulmer

Garra da falsa perna anal composta de 1 processo e 3 ou 4 pares de garras acessórias (Figs. 10, 21), cerda das garras tarsais não como acima ........................... 2

2(1). Garra da falsa perna anal com 1 processo e 4 pares de garras acessórias (Fig. 21); cerda das garras tarsais longa e fina (Fig. 20); abrigo de grãos de areia grossos com duas aberturas dorsais em forma de chaminé (Fig. 22) Protoptila Banks

Garra da falsa perna anal com 1 processo e 3 pares de garras acessórias (Fig. 10); cerda da garra tarsal curta e grossa (Fig. 9); abrigo com grãos de areia grossa com aberturas dorsais simples, sem chaminé (Fig. 11)

Itaura Müller

Chave de identificação para larvas de último estádio para os gêneros e subgênero de Hydropsychidae (modificada de Flint 1983)

1. Brânquias abdominais ventrais com haste central grossa, de onde se originam filamentos finos, distribuídos de forma uniforme (Figs. 29, 33, 36, 47)

Brânquias abdominais ventrais e ventro lateral sem haste central, bifurcando-se na base, com poucos filamentos grossos, distribuídos de maneira não uniforme (Fig. 41) . Smicridea McLahlan-2

2(1) Esclerito ventral do segmento abdominal VIII formado por placa única (Fig. 45) ....... Smicridea (Smicridea)

Esclerito ventral do segmento abdominal VIII formado por duas placas (Fig. 44). Smicridea (Rhyacophylax)

3(1). Trocantim largo na base, em forma de lâmina, terminando em um tufo de cerdas finas (Fig. 47) ou gancho; fêmur largo e truncado apicalmente; cabeça e tórax mais longos que largos (Figs. 47-49) ...... Synoestropsis Ulmer

Trocantim não alargado na base, pontiagudo apicalmente; fêmur não alargado e truncado apicalmente; cabeça e tórax não alongados como acima (Figs. 31, 30, 33, 37, 39) ... 4

4(3). Garra anal longa e fina (Fig. 33); cabeça ventralmente sem estrutura de estridulação (Fig. 35)

.. Macronema Pictet

Garra anal não alongada (Figs. 29, 32, 36); cabeça ventralmente com estrutura de estridulação (Figs. 30, 38)
5(4). Cabeça dorsalmente com carena (Figs. 36, 37); par de escleritos na base do labro (Fig. 37); abrigo de grãos de areia, parcialmente enterrado no sedimento, com duas aberturas, em forma de Y (Fig. 40) .......... Macrostemum Kolenati

Cabeça dorsalmente sem carena (Fig. 31); base do labro sem par de escleritos; abrigo de fragmentos vegetais, preso a rochas ou vegetação ........ Leptonema Guérin

Chave de identificação para larvas de último estádio para os gêneros de Hydroptilidae

1. Larva achatada dorso-ventralmente (Fig. 50) ou com abdome retrátil (Figs. 52, 57, 69, 100) ....................... 2

Larva não achatada dorso-ventralmente; abdome não retrátil (Figs. 62, 74, 79, 82, 89, 95).

2(1). Segmentos abdominais V e VI mais largos que o IV (Figs. 52, 57, 69, 100).

Segmentos abdominais IV, V e VI aproximadamente da mesma largura (Fig. 50) Alisotrichia Flint

3(2). Todos os segmentos abdominais com uma placa dorsal central (Figs. 52, 54, 69, 71, 72, 100, 101) ................. 4

Somente o segmento abdominal I com placa inteira (Fig. 58); segmentos II-VII sem placa dorsal, somente dois pequenos escleritos latero-dorsais (Figs. 57, 59); .......

Ceratotrichia Flint

4(3). Placas dorsais com duas lacunas circulares centrais (Figs. 100, 101); (Fig. 103) ........ Zumatrichia Mosely Placas dorsais sem lacuna circular central (Figs. 54, 71, 72) 5

5(4). Segmentos abdominais I-VII com esclerito com formas de meia lua ou sino com manchas escuras (Figs. 52, 54) Anchitrichia Flint

Segmentos abdominais I-VII com escleritos largos e curtos com coloração uniforme, sem manchas escuras (Figs. 69, 71, 72 . Leucotrichia Mosely

6(1). Falsas pernas anais, proeminente, destacando-se do abdome (Figs. 87, 95, 99) ... 7

Falsas pernas anais não se destacando do abdome (Figs. 66, 74, 89). 8

7(6). Cabeça longa e afilada, três vezes mais longa que larga, em forma de bico (Figs. 95, 96); sutura coronal pouco definida e a do frontoclípeo ausente (Fig. 96); dois pares de papilas digitiformes ventro-laterais no mesometatórax (Fig. 98) ........... Taraxitrichia Flint \& Harris

Cabeça não tão longa e afilada como acima; suturas coronal e do frontoclípeo presentes (Fig. 83); papilas ventro-laterais do abdome ausentes

Neotrichia Morton

8(6). Antenas com uma longa cerda mediana (Fig. 90); pernas anteriores mais curtas que aa médias e posteriores (Figs. 91-93); abrigo de seda transparente, com abertura estreitada anteriormente, como um gargalo de garrafa (Fig. 94) ......................... Oxyethira Eaton 
Antenas sem cerda mediana; pernas anteriores não como acima; abrigo de outra forma 9

9(8). Segmento abdominal IX com uma papila longa na base do esclerito dorsal e uma em cada esclerito da garra anal (Figs. 62, 66, 67); pernas recobertas por muitas cerdas; garras tarsais de todas as pernas com uma cerda na base (Figs. 63-65); abrigo bivalve de areia e algas (Fig. 68) ou somente areia .... Hydroptila Dalman Segmento abdominal IX sem papila ou apenas com uma papila no esclerito dorsal, sem papilas no esclerito lateral da garra anal; pernas não recobertas por muitas cerdas e somente garra do primeiro par de pernas com uma cerda na base (Figs. 75-77); abrigo de algas, com chaminé ou não (Figs. 78, 80) ou de fragmentos vegetais (Fig. 81) Metrichia Ross

Chave de identificação para larva de último estádio para os gêneros de Leptoceridae

1. Metanoto com três ou cinco escleritos (Figs. 115 e 119)

Metanoto membranoso ou com apenas dois pequenos escleritos (Figs. 107, 111, 125).

2(1). Metanoto com cinco escleritos (Fig. 115); pernas posteriores longas e finas, tíbia dividida em duas partes (Fig. 114); esclerito da gula longo e estreito, dividindo a gena (Fig. 116); abrigo feito com gravetos ocos (Fig. 117) .......... Triplectides egleri Sattler 1963

Metanoto com três escleritos (Fig. 119); pernas posteriores robustas, com a tíbia triangular achatada expandida lateralmente, não dividida (Fig. 123); esclerito anterior da gula pequeno e triangular (Fig. 121); abrigo de seda transparente (Fig. 118) ...... Amazonatolica hamadae Holzenthal \& Pes 2004

3(1). Metanoto totalmente membranoso (Fig. 111); palpo maxilar longo estendendo-se além do labro, (Fig. 110, 111); abrigo de areia (Fig. 112) ou de pequenos fragmentos vegetais sobrepostos de forma quadrangular (Fig. 113) .............. Oecetis McLachlan

Metanoto com dois pequenos escleritos ou manchas com cerdas (Figs. 107); palpo maxilar curto; abrigos de fragmentos vegetais com diferentes arranjos ou grãos de areia (Figs. 109, 128) ... 4

4(3). Placa dorsal do segmento abdominal IX e escleritos da garra anal com uma coroa de espinhos longos e fortes na margem posterior (Fig. 127); cabeça dorsalmente, com uma carena (Figs. 124, 125); esclerito da gula pequeno e triangular (Fig. 126) ................... gênero A

Placa dorsal do segmento abdominal IX e garra anal de outra forma sem coroa de espinhos (Fig. 108); cabeça dorsalmente, sem carena (Fig. 105); esclerito da gula grande, de forma trapezoidal (Fig. 106)

Nectopsyche Muller
Chave de identificação para larvas de último estádio para os gêneros de Philopotamidae

1. Margem anterior do frontoclípeo com reentrância (Fig. 135); cabeça com um par de cerdas pequenas e escuras localizadas na metade anterior da região ventral (Fig. 136) Chimarra Stephens

Margem anterior do frontoclípeo sem reentrância (Fig. 139); cabeça com um par de cerdas pequenas e escuras localizadas na metade posterior da região ventral (Fig. 140) Wormaldia McLachlan

Chave de identificação para larvas em último estádio para os gêneros de Polycentropodidae

1. Garra anal com um pente de dentes tão longos quanto a garra na região côncava (Figs. 151;155) Polyplectropus Ulmer

Garra anal sem pente de dentes longos (Fig. 144), se presentes são curtos (Figs. 146, 148) 2

2(1). Placa dorsal da falsa perna anal (entre a garra e o esclerito lateral) com duas faixas escuras que se tocam na região mediana formando um "X" (Fig. 145); garra anal sem dentes ou cerdas na região proximal (Fig. 144); cabeça com pequenas manchas escuras conspícuas (Fig. 143) Cernotina Ross

Placa dorsal da falsa perna anal (entre a garra e o esclerito lateral) com duas faixas escuras que não se tocam na região mediana (Fig. 149); garra da falsa perna anal com um par de dentes curtos (Fig. 148) ou cerdas na região proximal; cabeça sem manchas escuras conspícuas Cyrnellus Banks

\section{DISCUSSÃO}

Na família Xiphocentronidae, não foi possível detectar diferenças entre as larvas dos gêneros Machairocentron e Xiphocentron, a identificação foi realizada por meio da associação das larvas com o adulto farado. Os dois gêneros apresentam o mesmo comportamento, as larvas localizam-se sobre pedras, em áreas de remanso, ou fora da água em locais muito úmidos (Pes 2001).

As descrições das larvas do gênero Macrostemum (Wiggins 1996; Angrisano \& Korob 2001) indicam que as larvas desse gênero apresentam uma carena fechada na região posterior da cabeça. Entretanto, as larvas de Macrostemum sp., coletadas e associadas com adultos farados, não apresentam esta carena fechada e sim aberta como em Blepharopus diaphanus Kolenati, 1856 (Flint \& Wallace 1980).

Algumas diferenças observadas podem ser úteis para separar as larvas de Macrostemum sp. e B. diaphanus: a) a estrutura de estridulação na região ventral da cabeça de $B$. diaphanus, é marcada destacando-se em uma região mais clara, enquanto que em Macrostemum sp. esta região tem coloração uniforme; b) as larvas de Macrostemum sp. apresentam menos cerdas do que as de B. diaphanus; c) o abrigo de Macrostemum sp. é construído com areia, formando dois túneis em forma de 
"Y" para entrada e saída de água, a larva localiza-se na porção anterior que geralmente fica enterrada na areia (Fig. 40) ou troncos em decomposição, enquanto que em $B$. diaphanus o abrigo de areia ou/e fragmentos vegetais é simples, não tem esta forma.

Este é o primeiro registro da família Sericostomatidae para a região amazônica. Até o momento somente um gênero com uma espécie, Grumicha grumicha (Vallot) 1855, foi registrada na região sul do Brasil (Flint et al. 1999). A larva de Sericostomatidae coletada e ilustrada neste trabalho, claramente não pertence a esse gênero. A larva de Sericostomatidae gênero $\mathrm{A}$, é semelhante à larva de Notidobiella chacayana Schmid 1957, por apresentar carena dorsal na cabeça, muitas cerdas no tórax; ausência de escleritos laterais no mesotórax e abrigo de grãos de areia e seda (Flint 1967). Porém, pode pertencer a um gênero cuja larva não tenha sido descrita, como Chioecia Navas, Myotrichia Schmid (Flint et al. 1999), ou mesmo a um gênero novo. A identificação do gênero só será possível com a associação da larva com o adulto.

A presente chave é uma contribuição para o conhecimento dos Trichoptera na amazônia, porém muito ainda precisa ser feito, como revisões taxonômicas e levantamentos faunísticos para se conhecer quantas espécies ocorrem na região, além de realizar associações de larvas com os adultos descritos. São necessários ainda trabalhos sobre biologia, comportamento de larvas e adultos destes insetos para saber qual a sua importância biológica, o potencial bioindicador e seu papel na biologia de conservação na bacia amazônica.

Agradecimentos. A J. O. da Silva, J. Bosco, Y. B. Alencar e U. C. Barbosa (INPA) pelo auxílio nos trabalhos de campo. Aos funcionários da Secretaria de Turismo e Meio Ambiente do Município de Presidente Figueiredo pelo apoio no período das coletas. A Deyse C. Q. Santos e B. Otávio pela ilustração deste trabalho. À Capes pela concessão de bolsa de mestrado, ao CNPq pela concessão de bolsa de doutorado ao primeiro autor. Este trabalho foi financiado pelos PPI 1-3070, 1-3570 (INPA/ MCT), Edital Universal processo $n^{\circ} 479258 / 2001-5$ (CNPq) e Projeto Diversidade Biológica de Fragmentos Florestais (INPA/Smithsonian Institution), sub-projeto Ygarapés (série técnica $n^{\circ}$ : 438).

\section{REFERÊNCIAS}

Angrisano, E. B. 1995. Insecta Trichoptera, p. 1199-1237. In: E. C. Lopretto \& G. Tell (Eds.). Ecosistemas de aguas continentales, metodologia para su estudio. Tomo III. La Plata, Ediciones Sur, $1401 \mathrm{p}$

Angrisano, E. B. 1998. Trichoptera, p. 374-384. In: J. J. Morrone \& S. Coscarón (Eds.). Biodiversidad de Artrópodos Argentinos. La Plata, Ediciones Sur, 599 p.

Angrisano, E. B \& P. G. Korob. 2001. Trichoptera, p. 55-92. In: H. R. Fernández \& E. Domíngues (Eds.). Guía para la determinacíon de los artrópodos bentónicos sudamericanos. Tucumán, Universidad Nacional de Tucumán, 282 p.

Botosaneanu, L. \& O. S. Flint Jr. 1982. On some Trichoptera from Northern Venezuela and Ecuador (Insecta). Beaufortia 32: 1326.

Flint Jr., O. S. 1963. Studies of Neotropical Caddisflies, I: Rhyacophilidae and Glossosomatidae (Trichoptera). Proceedings of the United States National Museum 114: 453-478.

Flint Jr., O. S. 1967. Studies of Neotropical Caddisflies, II: Trichoptera collected by Prof. J. Illies in the Chilean Subregion. Beiträge zur
Neotropischen Fauna 5: 45-68.

Flint Jr., O. S. 1971. Studies of Neotropical Caddisflies, XII: Rhyacophilidae, Glossosomatidae, Philopotamidae, and Psycomyiidae from the Amazon Basin (Trichoptera). Amazoniana 3: $1-67$.

Flint Jr., O. S. 1974. Studies of Neotropical Caddisflies, XV: The Trichoptera of Surinam. Studies on the Fauna of Suriname and other Guyanas 14: $1-157$.

Flint Jr., O. S. 1978. Studies of Neotropical Caddisflies, XXII: Hydropsychidae of the Amazon Basin (Trichoptera). Amazoniana 6: $73-421$.

Flint Jr., O. S. 1981. Studies of Neotropical Caddisflies, XXVIII: The Trichoptera of the Río Limón. Smithsonian Contributions to Zoology 330: 1-61.

Flint Jr., O. S. 1983. Studies of Neotropical Caddisflies, XXXIV: The genus Plectromacromena (Trichoptera: Hydropsychidae). Proceedings of the Biological Society of Washington 96: 225-237.

Flint Jr., O. S. 1991. Studies of Neotropical Caddisflies, XLIV: On a collection from Ilha de Maraca, Brazil. Acta Amazônica 21: $63-$ 83.

Flint Jr., O. S. 1996. The Trichoptera collected on the expeditions to Parque Manu, Madre de Dios, Peru. In: D. E. Wilson \& A. Sandoval (Eds). Manu: the biodiversity of southeastern Peru. Washington, Smithsonian Institution Press, 679 p.

Flint Jr., O. S. \& J. Bueno-Soria. 1982. Studies of Neotropical Caddisflies, XXXII: The immature stages of Macronema viriipenne Flint \& Bueno, with the division Macronema by the resurrection of Macrostemum (Trichoptera: Hydropsychidae). Proceedings of the Biological Society of Washington 95: $358-370$.

Flint Jr., O. S. \& S. C. Harris. 1992. Studies of Neotropical Caddisflies, XLII: Taraxitrichia amazonensis, a new genus and species of microcaddisfly from Venezuela (Trichoptera: Hydroptilidae), $\mathrm{p}$ 411-414. In: C. Tomaszewski (Ed.). Proceedings of the Sixth International Symposium on Trichoptera. Lódz-Zakopane, Adam Michiewicz University Press, xiv+479 p.

Flint Jr., O. S. \& L. A. Reyes. 1991. Studies of Neotropical Caddisflies, XLVI: The Trichoptera of the Rio Moche Basin, Department of la Libertad, Peru. Proceedings of the Biological Society of Washington 104: 474-492.

Flint Jr., O. S. \& J. B. Wallace. 1980. Studies of Neotropical Caddisflies, XXV: The immature stages of Blepharopus diaphanus and Leptonema columbianus (Trichoptera: Hydropsychidae). Proceedings of the Biological Society of Washington 93: $178-193$.

Flint Jr., O. S.; S. C. Harris, \& L. Botosaneanu. 1994. Studies of Neotropical Caddisflies, L: The description of Cerasmatrichia, new genus, a relative of Alisotrichia, with descriptions of new and old species and the larva (Trichopera: Hydroptilidae). Proceedings of the Biological Society of Washington 107: 360-382.

Flint Jr., O. S.; R.w. Holzenthal \& S. C. Harris. 1999. Catalog of the Neotropical Caddisflies (Insecta: Trichoptera). Columbus, Ohio Biological Survey, iv $+239 p$.

Guahyba, R. R. 1991. Estágios imaturos de Anchitrichia duplifurcata Flint, 1983 (Trichoptera, Hydroptilidae). Revista Brasileira de Biologia 35: 121-125.

Holzenthal, R. W. 1995. The caddisfly genus Nectopsyche: new gemma group species from Costa Rica and the Neotropics (Trichoptera: Leptoceridae). Journal of the North American Benthological Society 14: 61-83.

Holzenthal, R. W. \& A. M. O. Pes. 2004. A new genus of long-horned caddisfly from the Amazon basin (Trichoptera: Leptoceridae: Grumichellini). Zootaxa 621: 1-16.

Marlier, G. 1964. Trichoptères de l'Amazonie recueillis par Professeur H. Sioli. Memoires Institut Royal des Sciences naturalles de Belgique, series 2, fasc. 76: $1-167$.

Martynov, A. B. 1912. On two collections of Trichoptera from Peru. Annuaire de Musée Zoologique de l'Académie Impérial des Sciences de St. Pétersbourg 17: 1-40.

Pes, A. M. O. 2001. Taxonomia e estrutura de comunidade de Trichoptera (Insecta) no município de Presidente Figueiredo, Amazonas, Brasil. Dissertação de Mestrado. Manaus, Instituto Nacional de Pesquisas da Amazônia, 164 p.

Pes, A. M. O. \& N. Hamada. 2003. The occurrence of Taraxitrichia Flint \& Harris, 1992 (Trichoptera: Hydroptilidae) in Brazil, with 
description of the final larval stage. Zootaxa 328: 1-7.

Pes, A. M. O. \& N. Hamada. 2004. Ceratotrichia Flint, 1992 (Trichoptera: Hydroptilidae) larval and pupal description and new genus records for Brazil. Entomotropica 19: 31-37.

Prather, L. A. 2003. Revision of the Neotropical caddisfly genus Phylloicus (Trichoptera: Calamoceratidae). Zootaxa 275: 1-214.

Resh, V. H \& J. O. Solem. 1996. Phylogenetic Relationships and Evolutionary adaptations of aquatic insects. In.: R. W. Merritt \& K. W. Cummins (Eds). An Introdution to the Aquatic Insects of North America. 3rd Ed. Dubuque, Kendall/Hunt Publishing Company, 862 p.

Roback, S. S. 1966. The Trichoptera larvae and pupae. p. 235-303. In: R. Patrick; F. A. Aldrich; J. Cairns Jr.; F. Drouet; M. H. Hohn; S. S. Roback; H. Skuja; P. J. Spangler, Y. H. Swabey \& L. A. Whitford (Eds.). Catherwood Foundation Peruvian-Amazon expedition. Monographs of the Academy of Natural Sciences of Philadelphia 14: 1-495.

Sattler, W. 1963a. Eine neue Triplectides - Art (Leptoceridae,
Trichoptera) aus dem brasilianischen Amazonasgebeit, ihre Metamorphosestadien und Bemerkungen zu ihrer Biologie. Beiträge zur Neotropische Fauna 3: 20-33.

Sattler, W. 1963b. Über den Körperbau, die Ökologie und Ethologie der Larve und Puppe von Macronema Pict. (Hydropsychidae), ein als Larve sich von "Mikro-Drift"ernährendes Trichopter aus dem Amazonasgebiet. Stuttgart. Archiv Für Hydrobiologie 59: 2660.

Sattler, W. \& J. Sykora 1977. Über eine durch ihr Bauinstinkt, merkwürdige neotropische Köcherflie - Leucotrichia brasiliana $\mathrm{n}$. sp. (Trichoptera, Hydroptilidae). Amazoniana 6: 237-255.

Ulmer, G. 1905. Zur Kenntniss aussereupäischer Trichopteren. Stettiner Entomologische Zeitung 66: 1-119.

Ulmer, G. 1906. Ueber die Larve einer Brasilianischen Trichopteren Spezies. Annales de Biologie Lacustre 1: 1-8.

Wiggins, G. B. 1996. Larvae of the North American Caddisfly genera (Trichoptera). 2rd Ed. Toronto, University of Toronto Press Incorporated, $457 \mathrm{p}$.

\section{APÊNDICE}
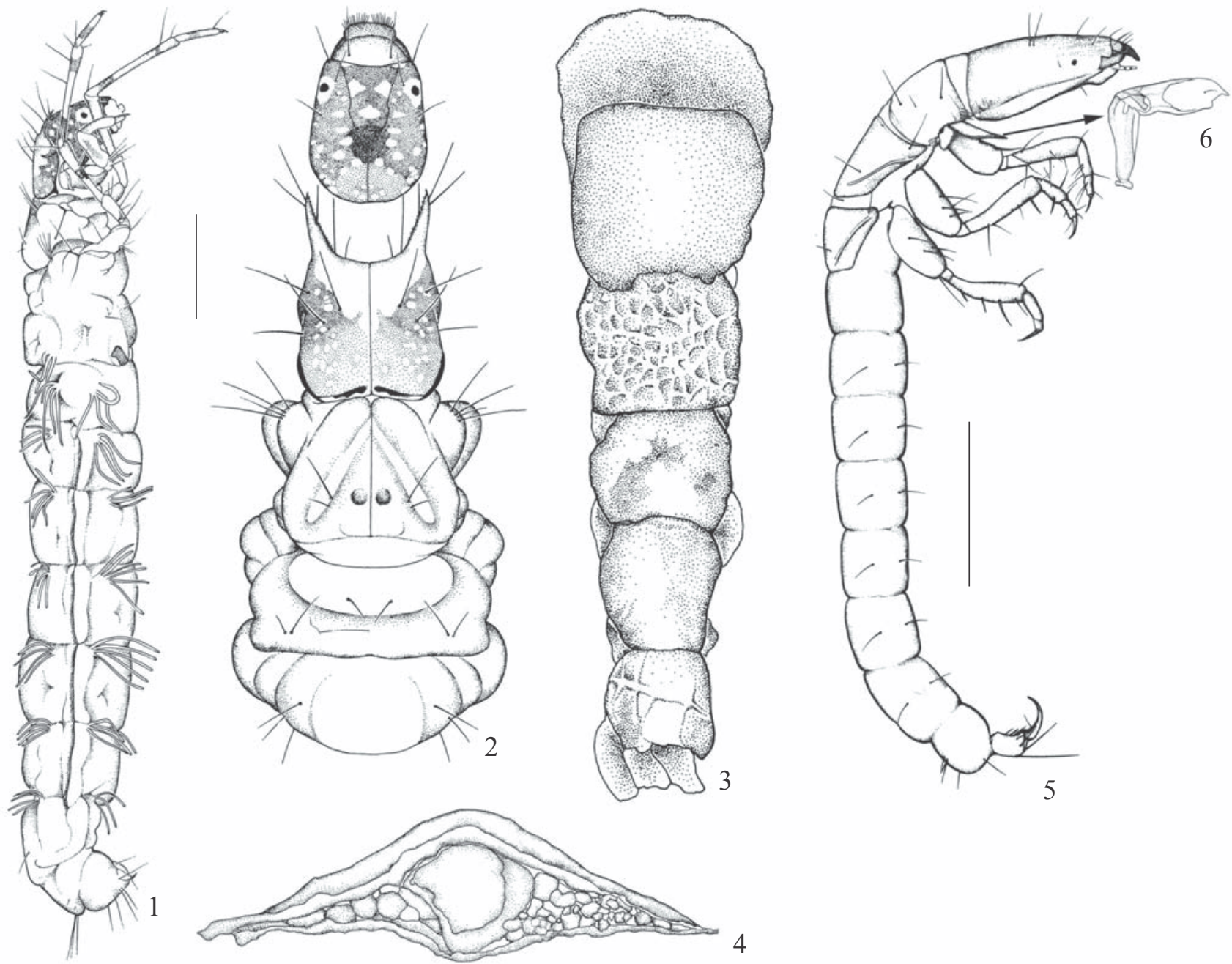

Figs. 1-6. 1-4, Calamoceratidae. Phylloicus sp. 2; 1, larva vista lateral; 2, cabeça e tórax vista dorsal; 3, abrigo vista ventral; 4, abrigo corte transversal. Escala $=2 \mathrm{~mm}$.; 5-6, Ecnomidae. Austrotinodes sp. 5, larva vista lateral; 6, trocantim vista ventral. Escala $=1 \mathrm{~mm}$. 

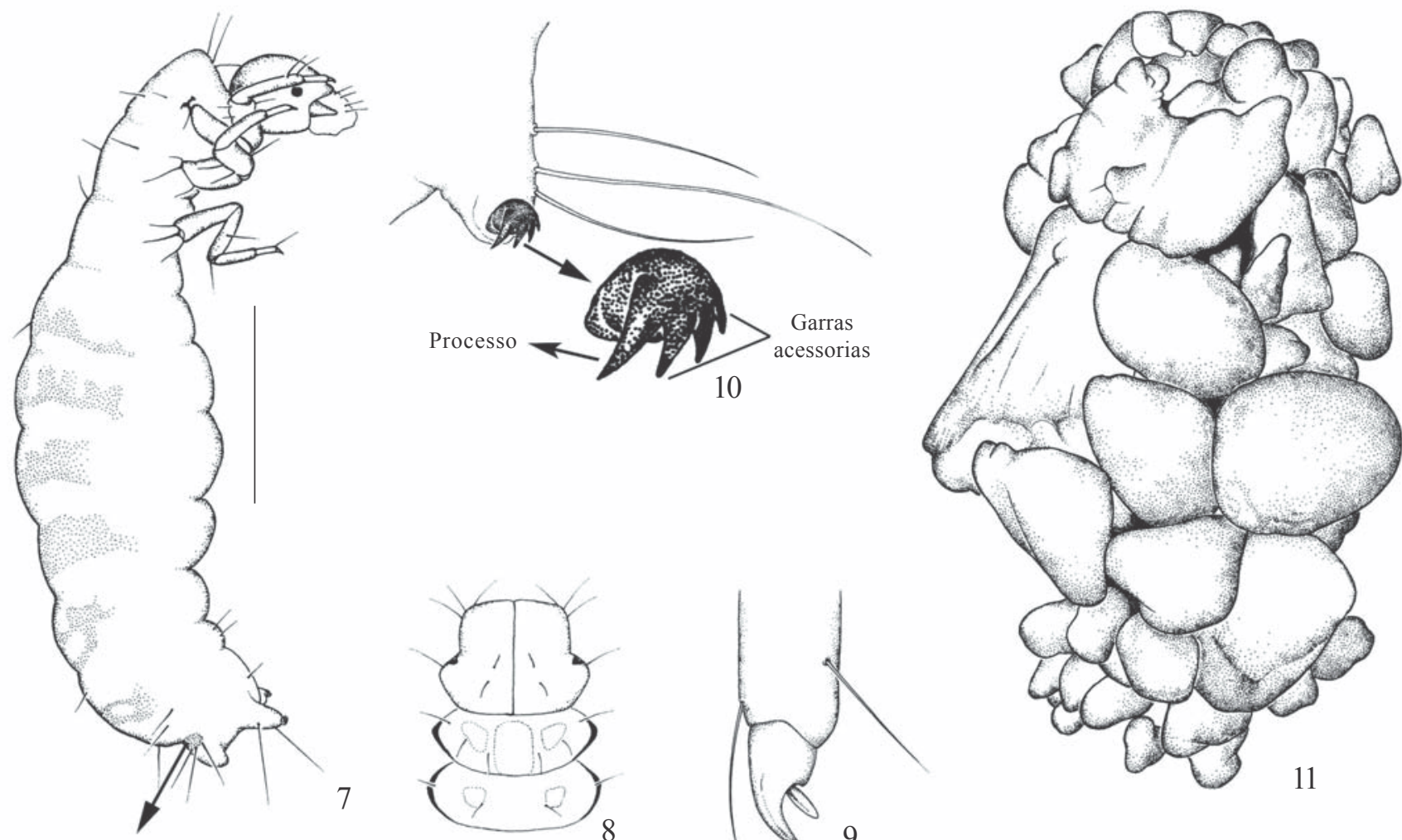

Esclerito IX segmento
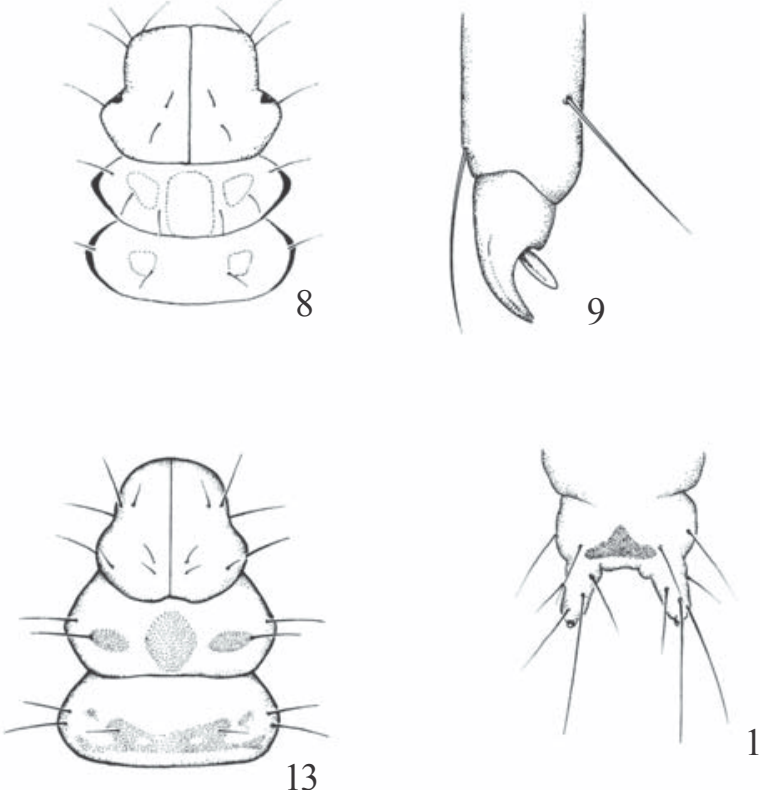

14

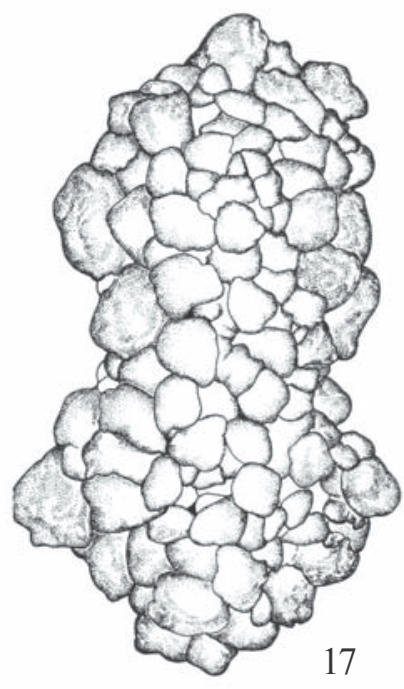

12

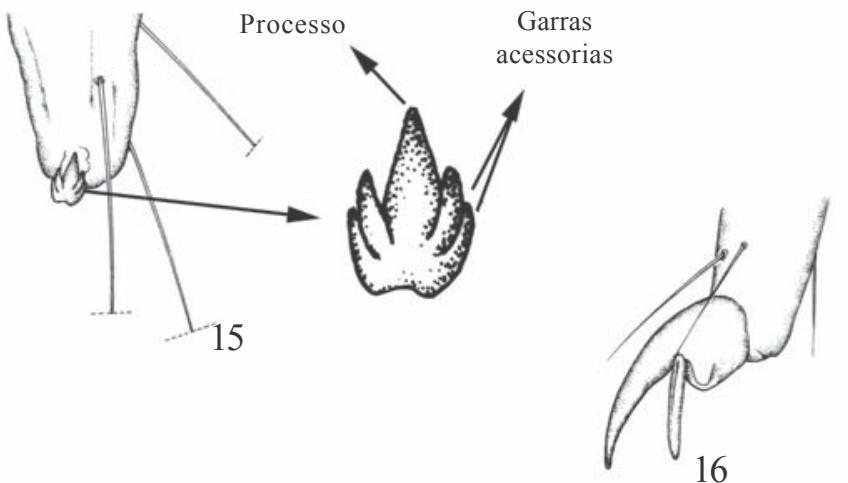

17

Figs. 7-17. Glossosomatidae. 7-11, Itaura sp. 7, larva vista lateral; 8, tórax vista dorsal; 9, garra tarsal do primeiro par de pernas; 10, garra da falsa perna anal (ampliação, mostrando processo e garras acessórias); 11, abrigo vista dorsal. 12-17. Mortoniella sp. 1. 12, larva vista lateral; 13, tórax vista dorsal; 14, segmento abdominal IX vista dorsal; 15, garra da falsa perna anal (ampliação, mostrando processo e garras acessórias); 16, garra tarsal da perna anterior; 17 , abrigo vista dorsal. Escala $=1 \mathrm{~mm}$. 

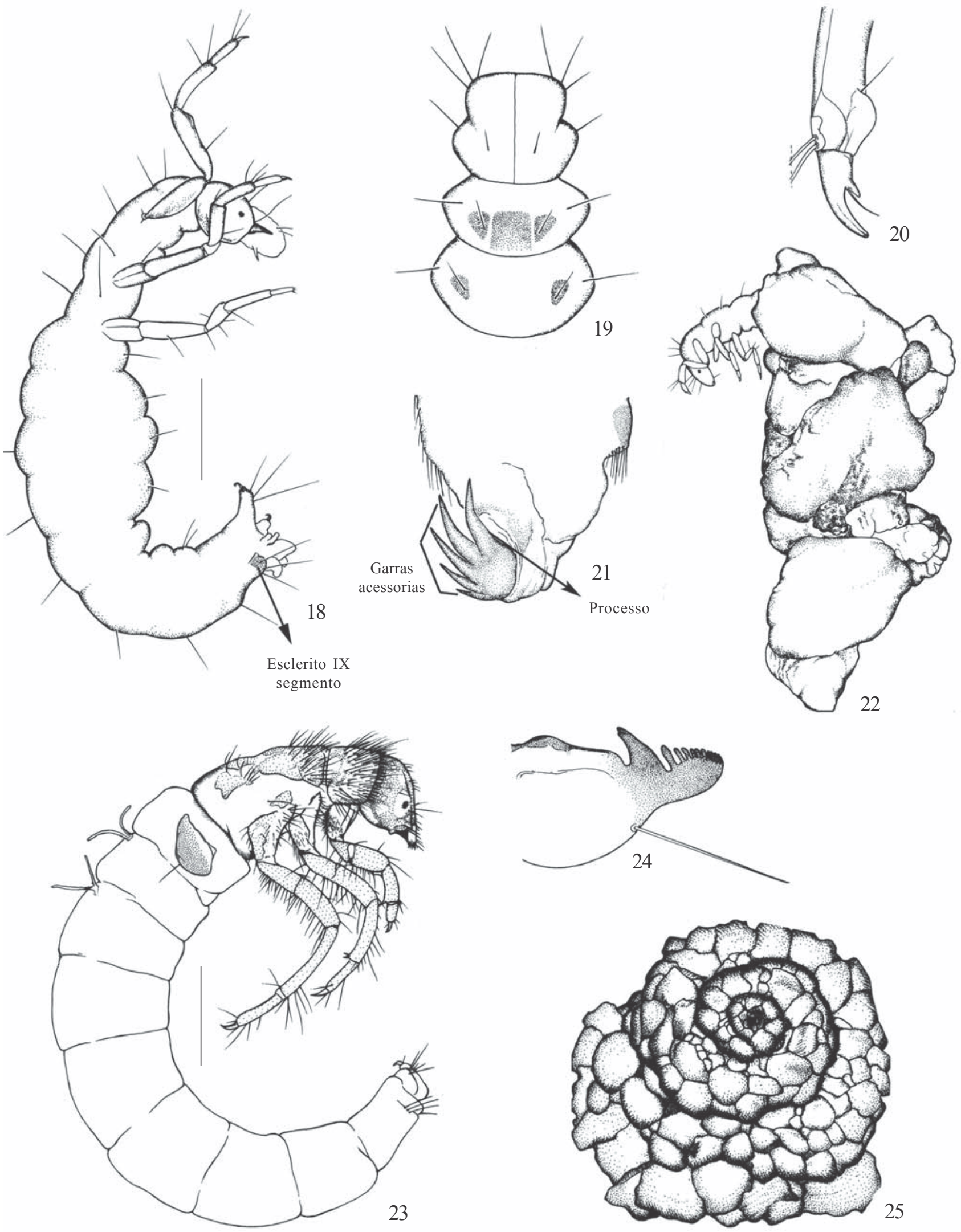

Figs. 18-25. 18-22, Glossosomatidae. Protoptila sp. 18, larva vista lateral; 19, tórax vista dorsal; 20, garra tarsal da perna anterior; 21, garra da falsa perna anal; 22, abrigo vista lateral. Escala $=0,5 \mathrm{~mm}$. 23-25, Helicopsychidae. Helicopshyche sp. 23, larva vista lateral; 24, garra da falsa perna anal; 25, abrigo vista dorsal. Escala $=2 \mathrm{~mm}$. 

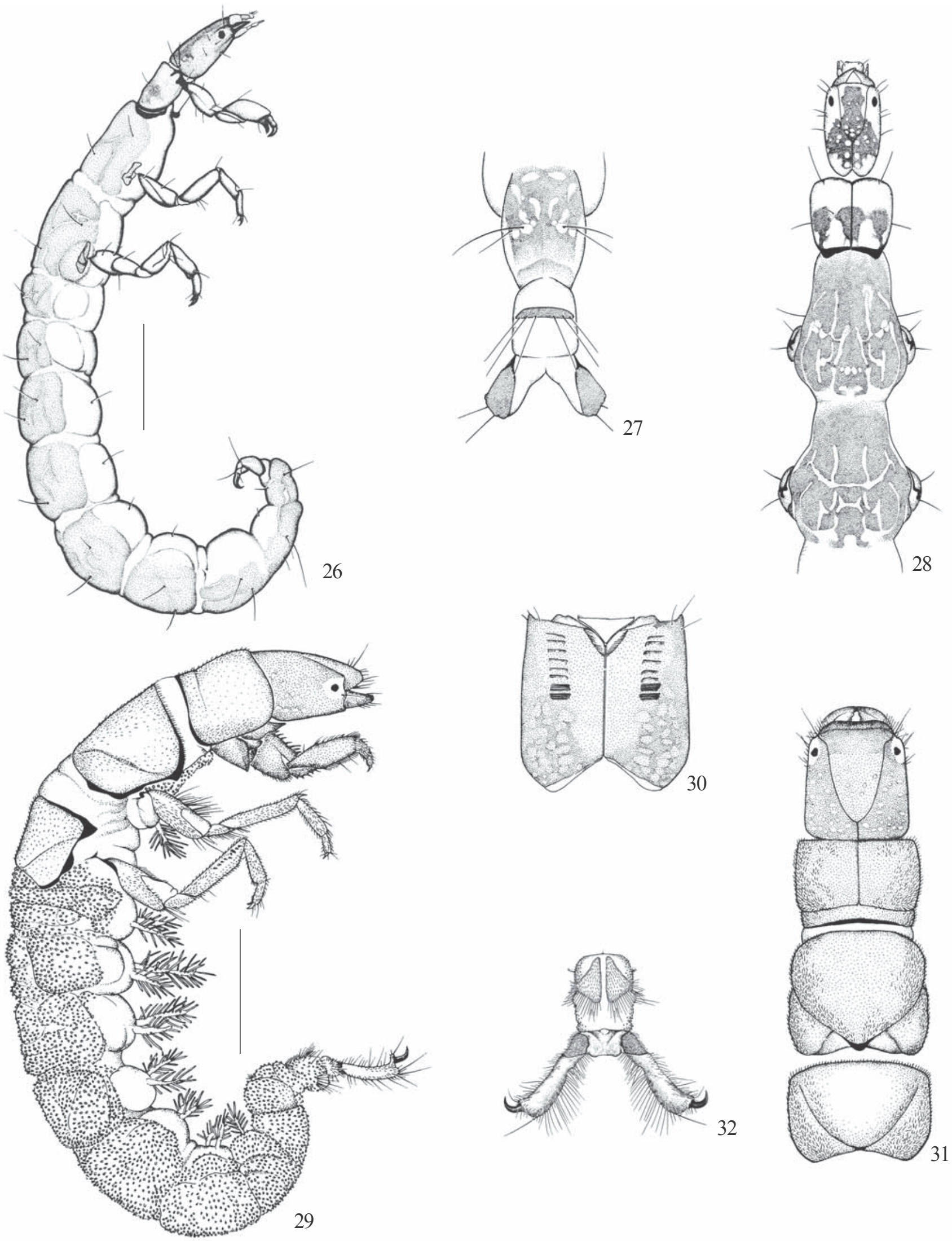

Figs.26-32. 26-28, Hydrobiosidae. Atopsyche sp. 26, larva, vista lateral; 27, segmentos abdominais VIII e IX e esclerito da falsa perna anal vista dorsal; 28, tórax vista dorsal. 29-32, Hydropsychidae. Leptopnema sp. 3. 29, larva vista lateral; 30, cabeça vista ventral; 31, cabeça e tórax vista dorsal; 32, segmento abdominal IX e garra da falsa perna anal vista ventral. Escala $=2 \mathrm{~mm}$. 

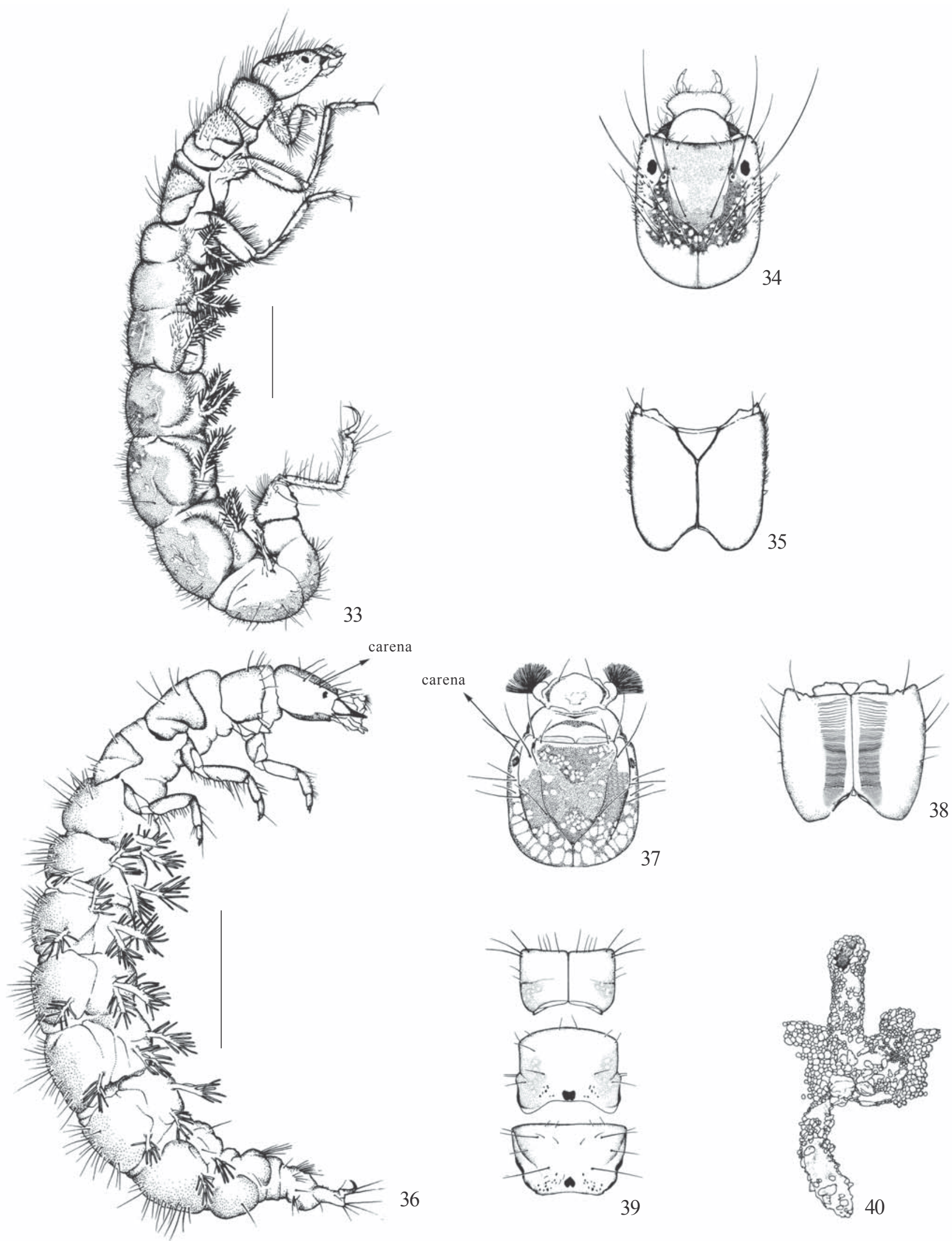

39

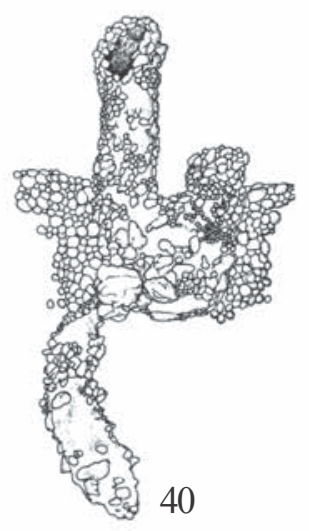

Figs. 33-40. Hydropsychidae. 33-35, Macronema sp.1. 33, larva vista lateral; 34, cabeça vista dorsal; 35, cabeça vista ventral. 36-40, Macrostemum sp. 36, larva vista lateral; 37, cabeça vista dorsal; 38, cabeça vista ventral; 39, tórax vista dorsal; 40, abrigo. Escala $=2 \mathrm{~mm}$. 

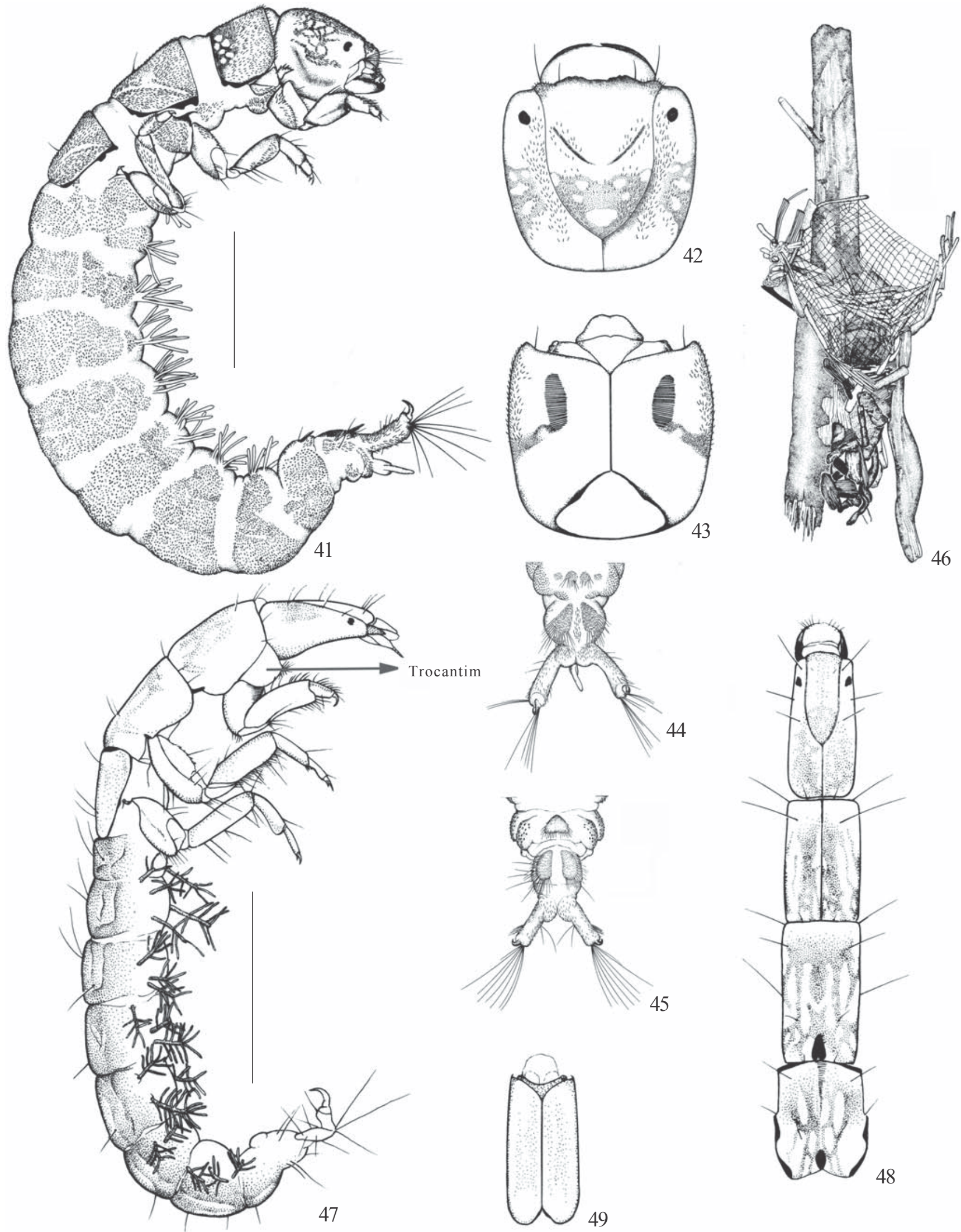

Figs. 41-49. Hydropsychidae. 41-46, Smicridea (Rhyacophilax) sp. 2. 41, larva vista lateral; 42, cabeça vista dorsal; 43, cabeça vista ventral; 44, segmentos abdominais VIII e IX e falsas pernas anais. Smicridea (Smicridea) sp. 3. 45, segmentos abdominais VIII e IX e falsas pernas anais; 46, abrigo. 47-49. Synoestropsis sp. 47, larva vista lateral; 48, cabeça e tórax, vista dorsal; 49, cabeça vista ventral. Escala $=2 \mathrm{~mm}$. 

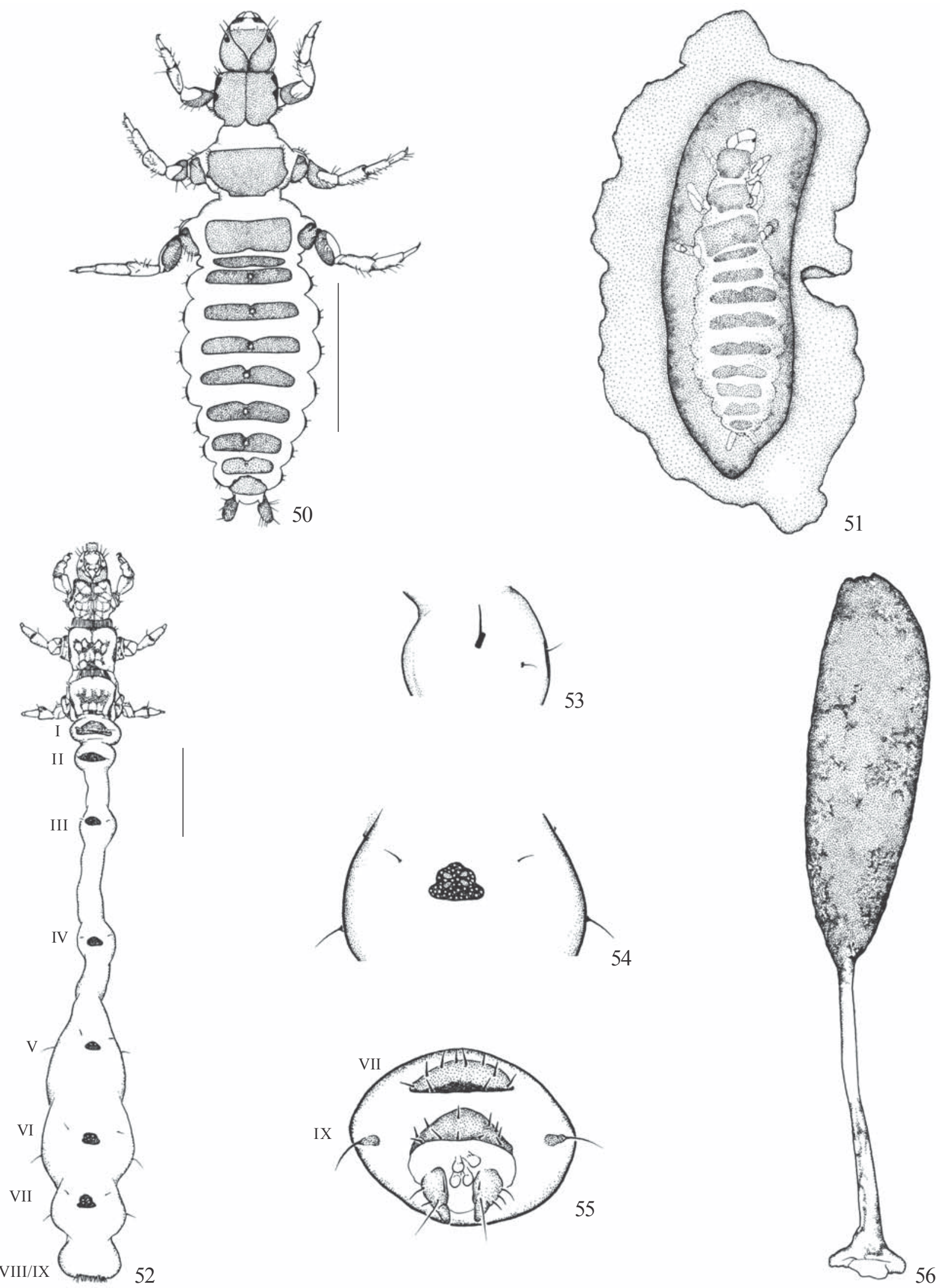

Figs. 50-56. Hydroptilidae. 50-51, Alisotrichia sp. 50, larva vista dorsal; 51, abrigo da pupa (por transparência larva pré-pupa). 52-56, Anchitrichia sp. 52, larva, vista dorsal; 53, esclerito lateral do segmento abdominal III; 54, esclerito dorsal do segmento abdominal V; 55, vista dorso posterior dos segmentos abdominais VIII e IX; 56, abrigo. Escala $=1 \mathrm{~mm}$. 

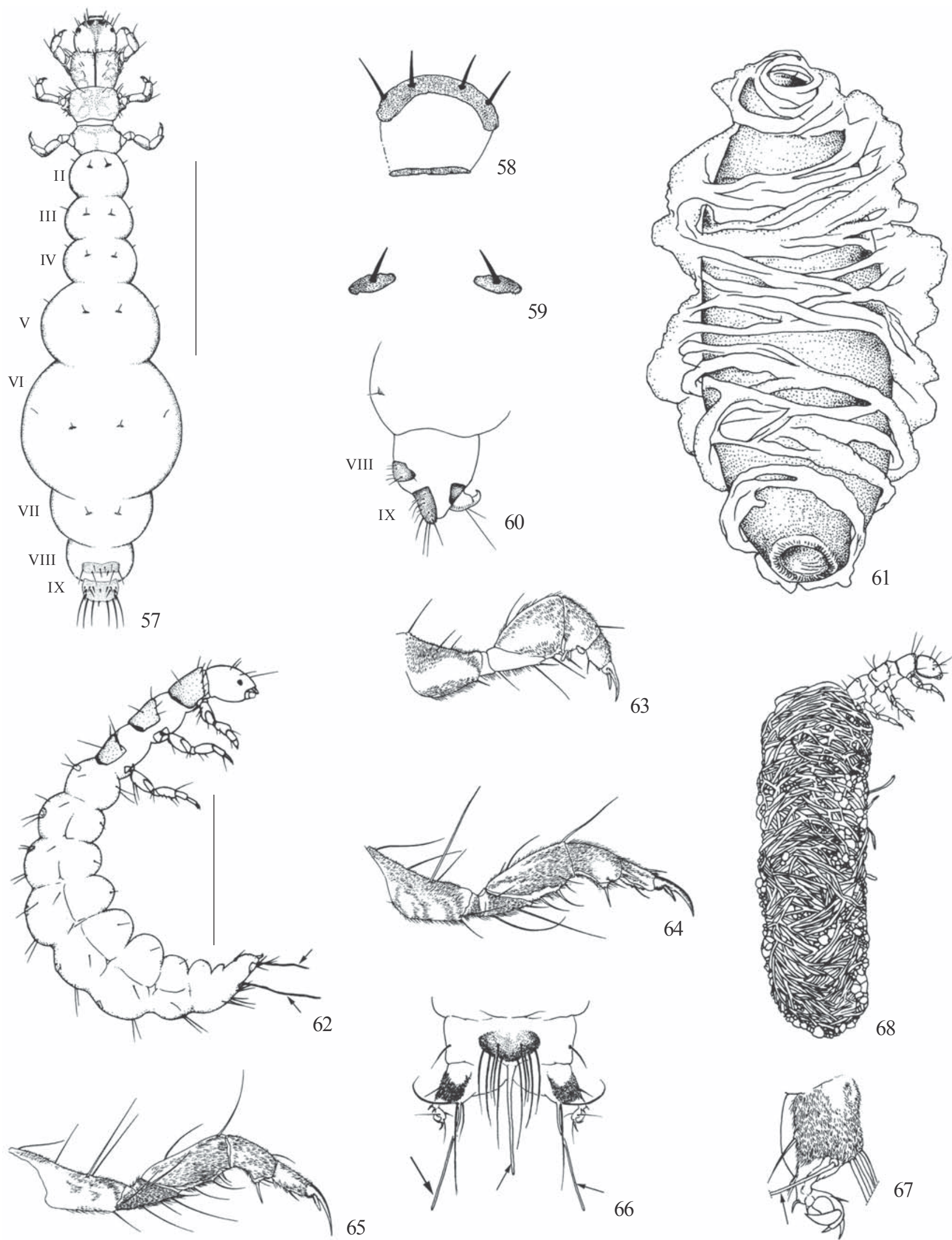

65
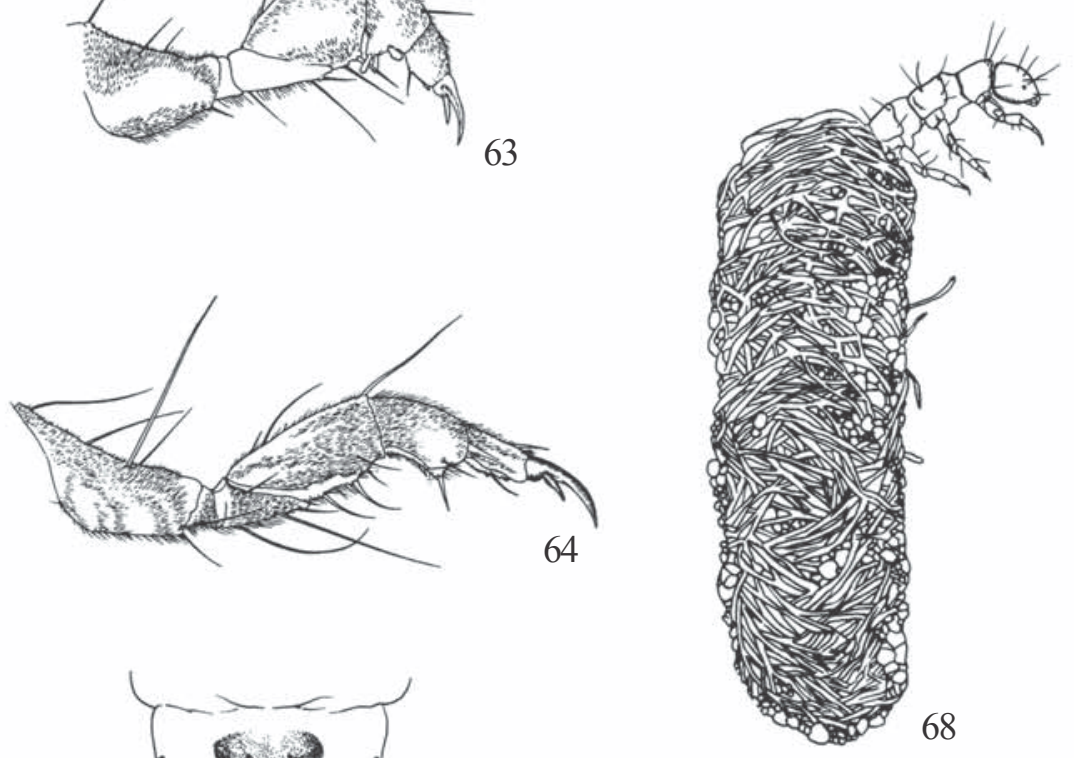

Figs. 57-68. Hydroptilidae. 57-61, Ceratotrichia sp. 57, larva vista dorsal; 58, escleritos dorsais do segmento abdominal I; 59, esclerito dorsal dos segmentos abdominais II-VII; 60, vista lateral dos segmentos abdominais VIII e IX; 61, abrigo. 62-68, Hydroptitila sp. 62, larva vista lateral; 6365, primeiro, segundo e terceiro pares de pernas; 66, segmento abdominal IX e falsas pernas anais, vista dorsal; 67, garra da falsa perna anal; 68, abrigo. (Setas indicando papilas do esclerito dorsal e garra anal). Escala $=1 \mathrm{~mm}$. 


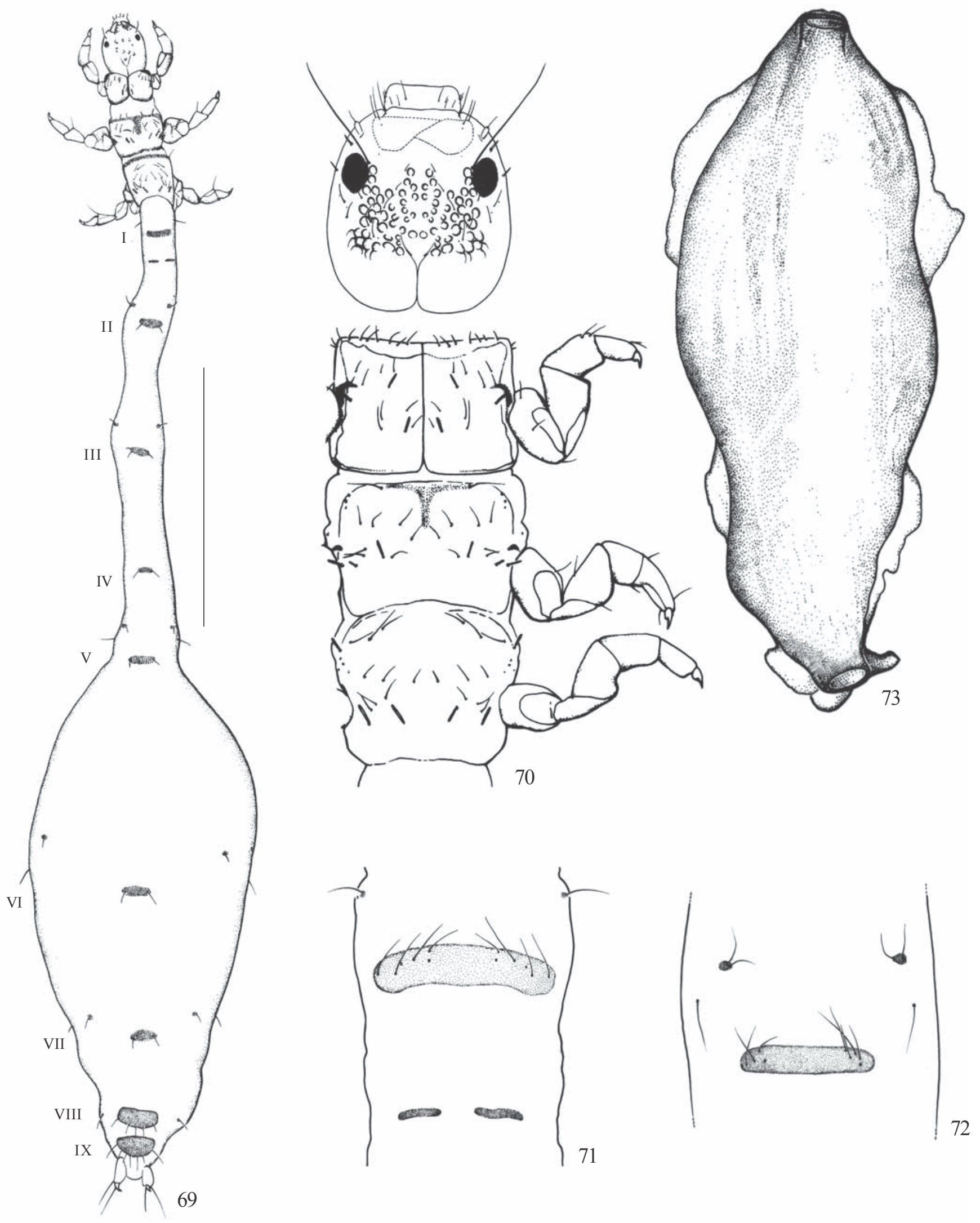

Figs. 69-73. Hydroptilidae Leucotrichia sp. 69, larva vista dorsal; 70, cabeça e tórax vista dorsal; 71, escleritos dorsais do segmento abdominal I; 72, escleritos dorsais do segmento abdominal II; 73. abrigo. Escala $=1 \mathrm{~mm}$. 

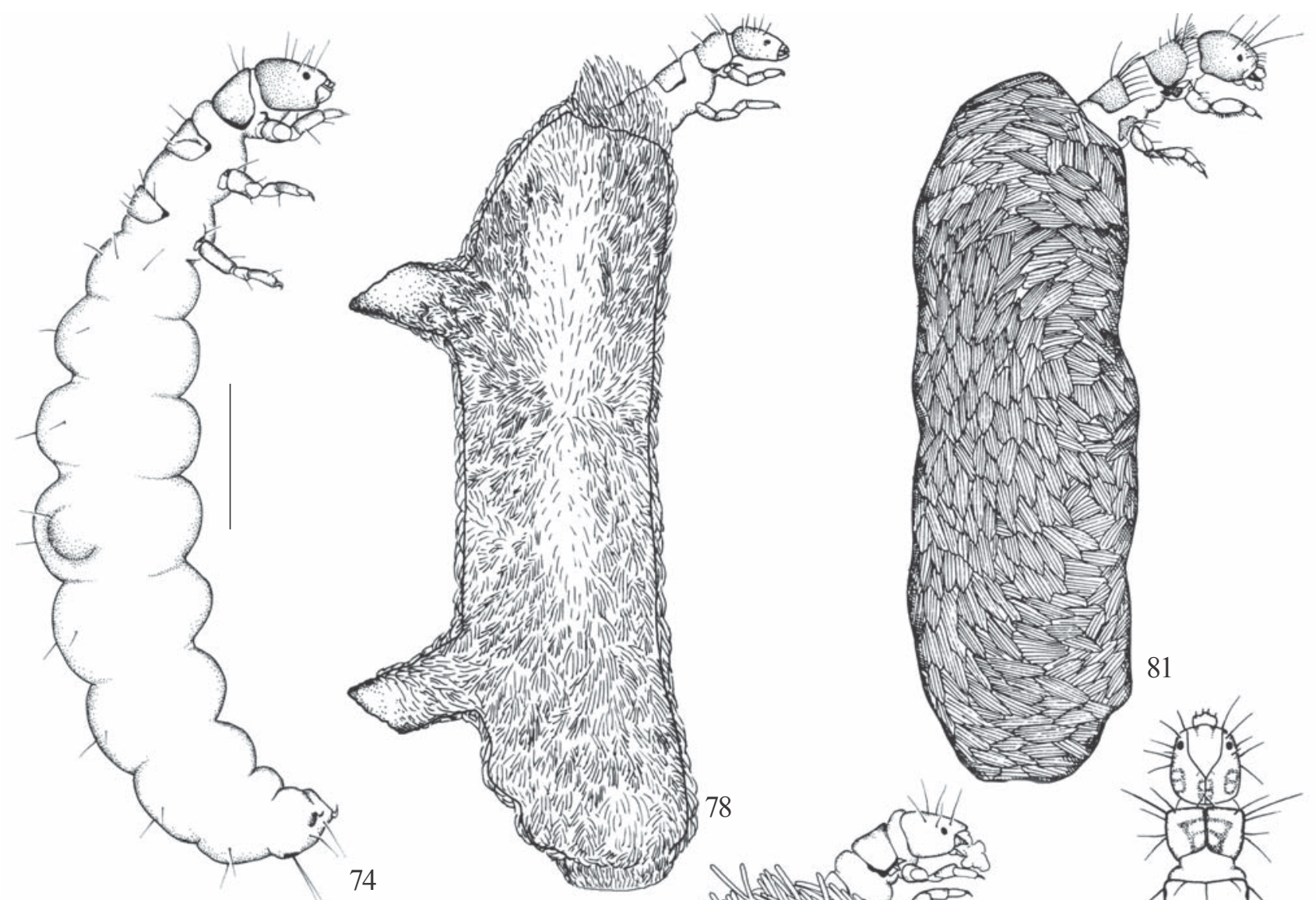

74
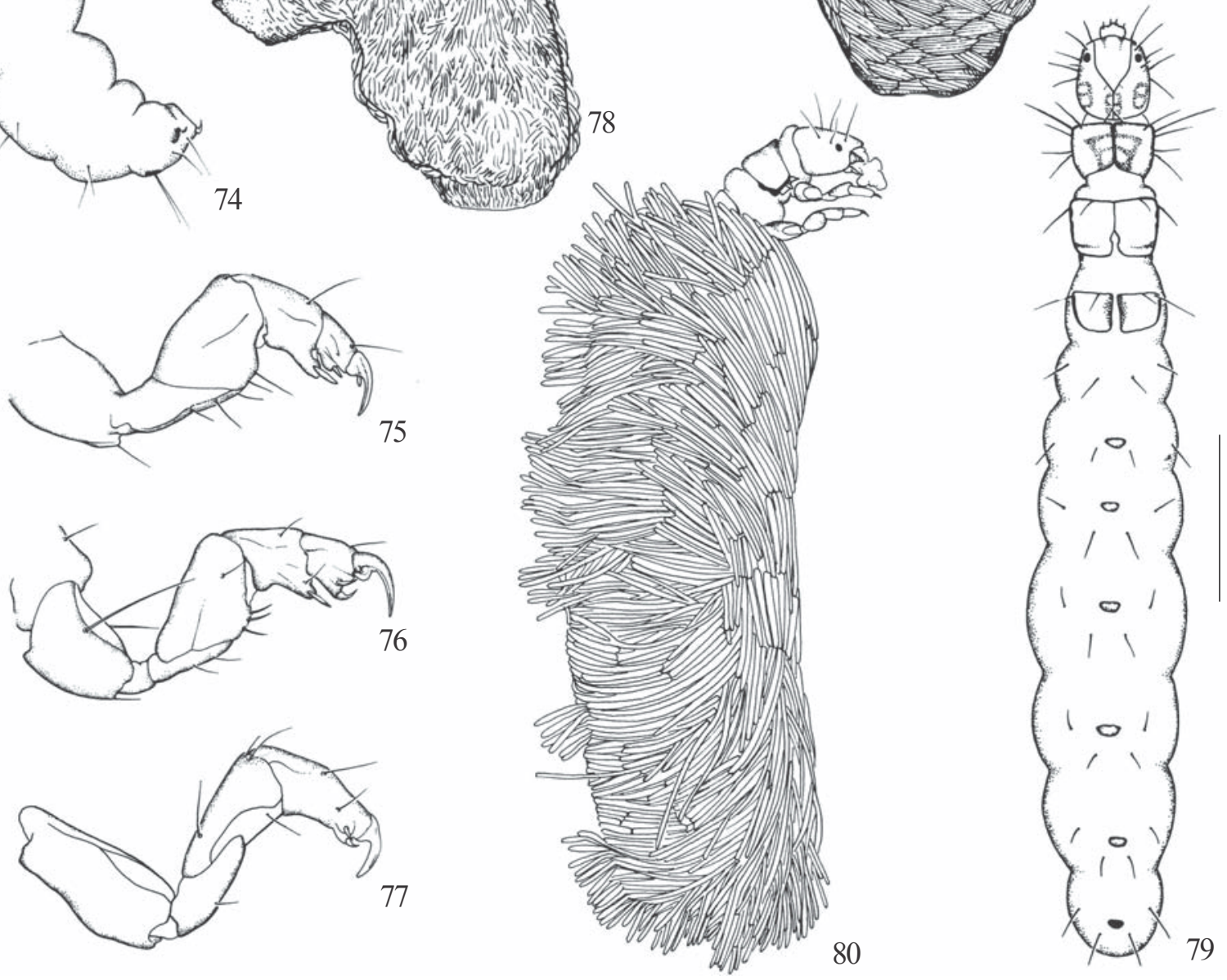

Figs. 74-81. Hydroptilidae Metrichia sp. 1. 74, larva vista lateral; 75-77, primeiro, segundo e terceiro pares de pernas; 78, abrigo. Metrichia sp. 2. 79, larva vista dorsal; 80, abrigo. Metrichia sp. 3. 81, abrigo. Escala $=1 \mathrm{~mm}$. 


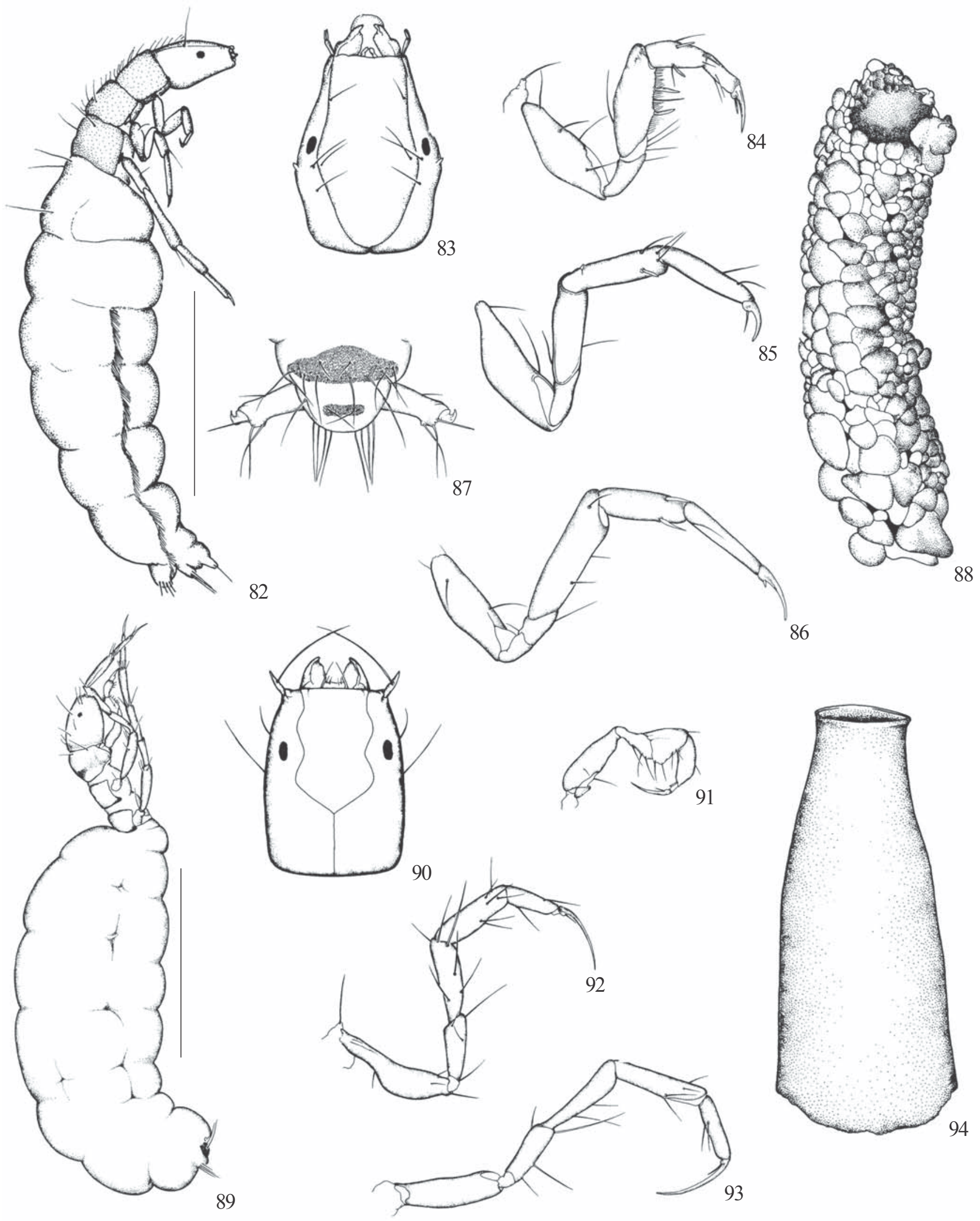

Figs. 82-94. Hydroptilidae. 82-88, Neotrichia sp. 82, larva vista lateral; 83. cabeça vista dorsal; 84-86, primeiro, segundo e terceiro pares de pernas; 87, segmento abdominal IX e falsas pernas anais vista dorsal; 88, abrigo. 89-94, Oxyethira sp. 89, larva vista lateral; 90, cabeça vista dorsal; 91-93, primeiro, segundo e terceiro pares de pernas; 94, abrigo. Escala $=1 \mathrm{~mm}$. 

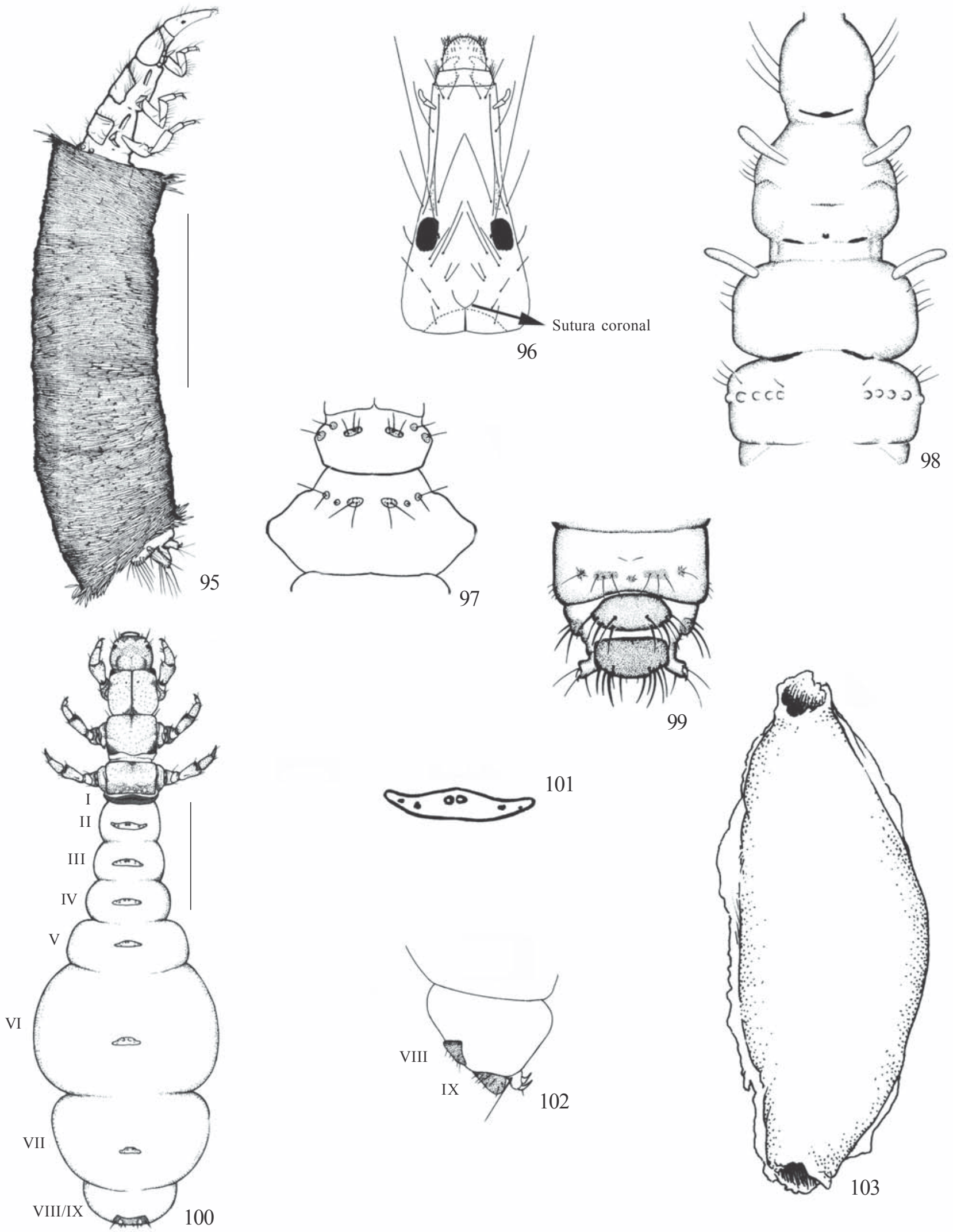

96
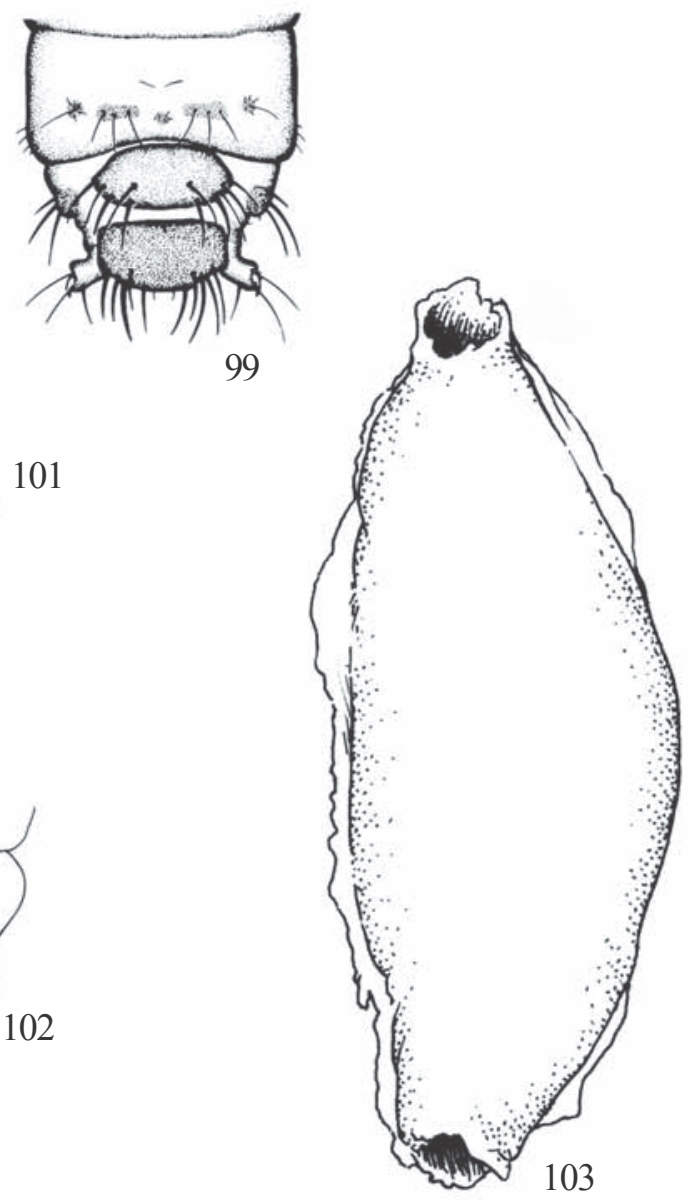

Figs. 95-103. Hydroptilidae. 95-99, Taraxitrichia sp. 95, abrigo e larva vista lateral; 96, cabeça dorsal; 97, segmentos abdominais I e II vista dorsal; 98, tórax e segmento abdominal I vista ventral; 99, segmentos abdominais VIII e IX e falsas pernas anais. (Figs. 96, 98 adaptadas de Pes \& Hamada 2004). 100-103, Zumatrichia sp. 100, larva vista dorsal; 101, esclerito dorsal do segmento abdominal II; 102, vista lateral dos segmentos abdominais VIII e IX; 103, abrigo. Escala $=1 \mathrm{~mm}$. 

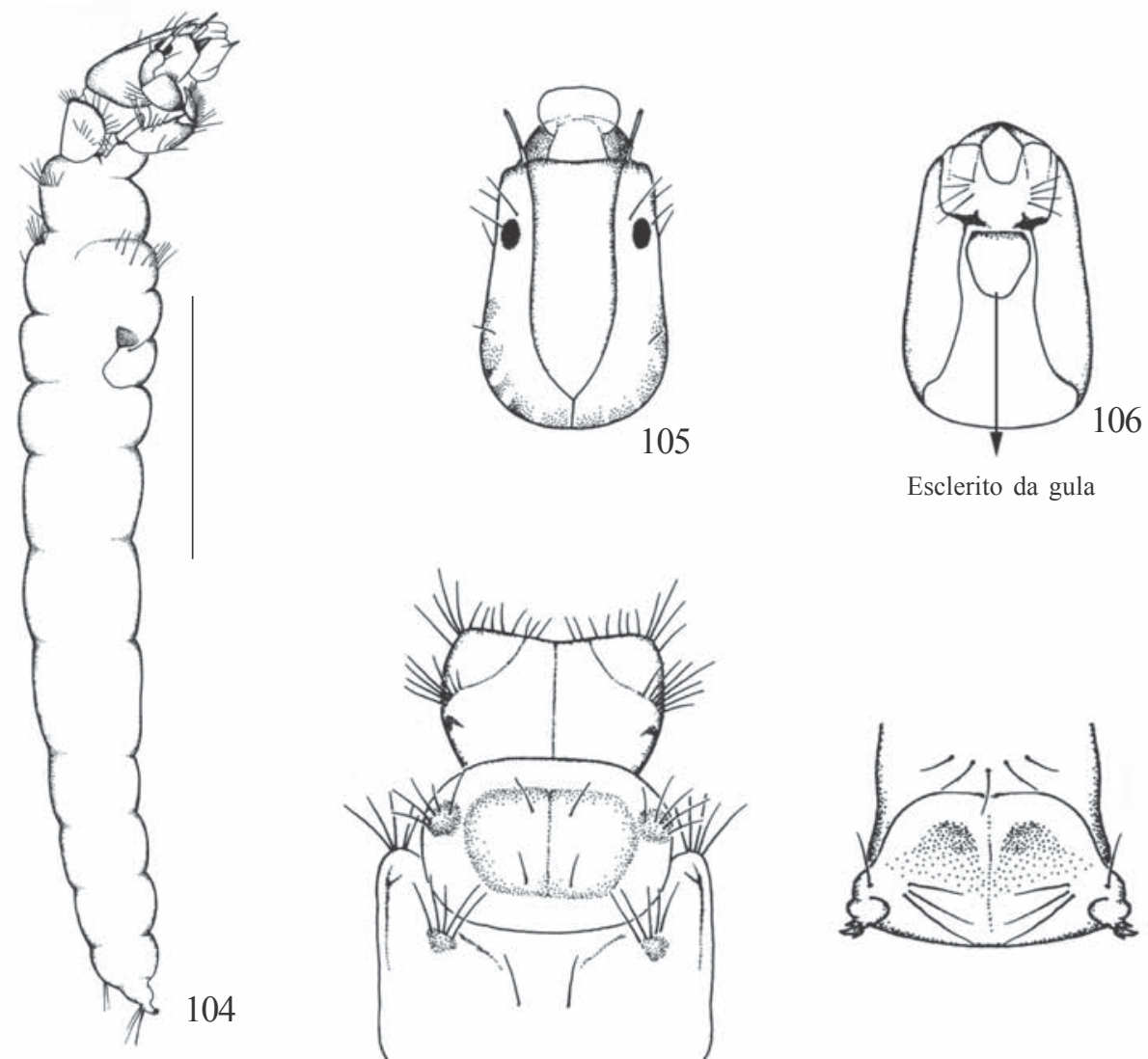

Esclerito da gula
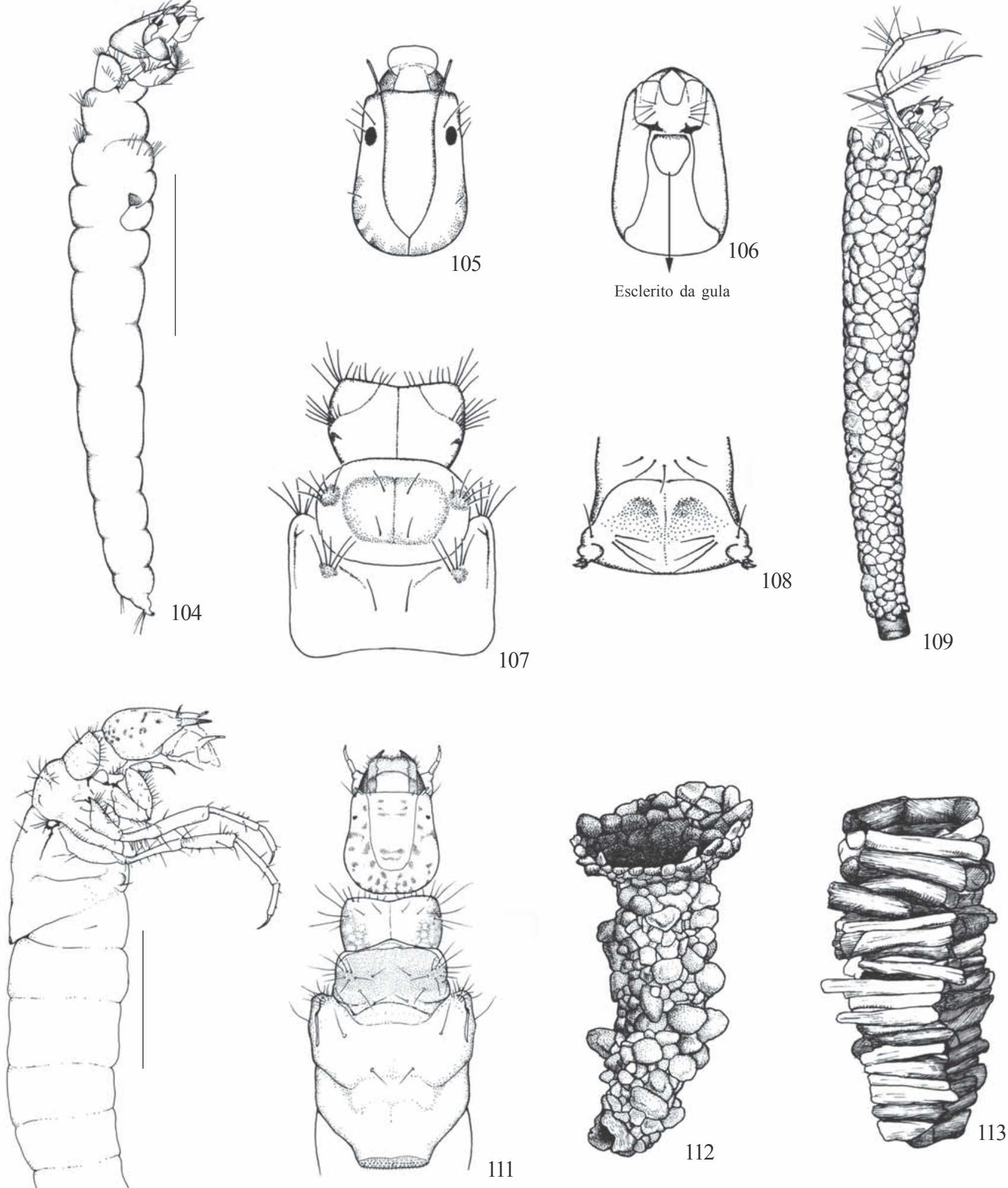

Figs. 104-113. Leptoceridae. 104-109, Nectopsyche sp. 104, larva vista lateral (somente com o primeiro par de pernas); 105, cabeça vista dorsal; 106, cabeça vista ventral; 107, tórax vista dorsal; 108, segmento abdominal IX vista ventral; 109, abrigo. 110-113, Oecetis sp. 1. 110, larva vista lateral; 111, cabeça e tórax vista dorsal; 112, abrigo. Oecetis sp. 2. 113, abrigo. Escala $=1 \mathrm{~mm}$. 

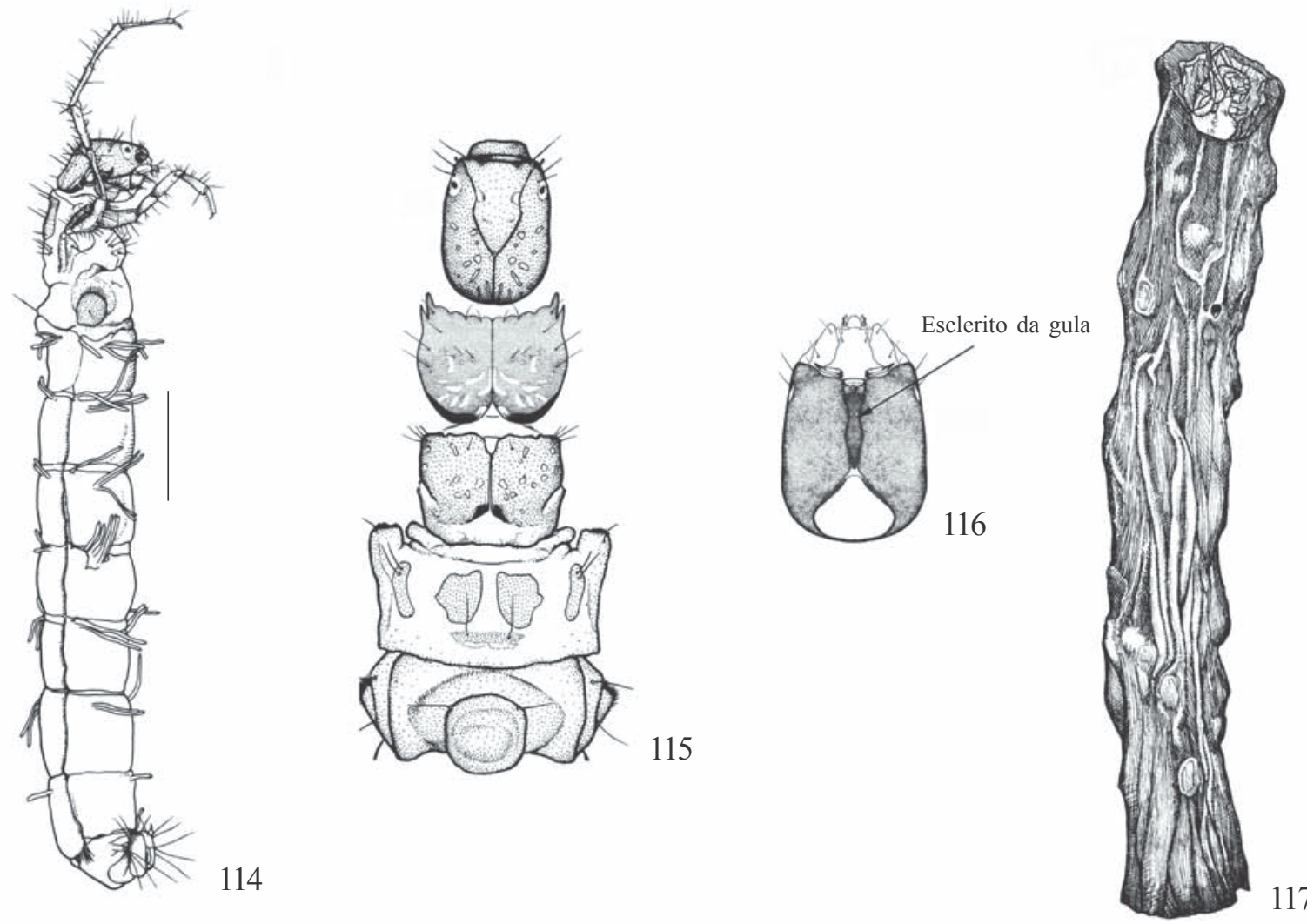

115
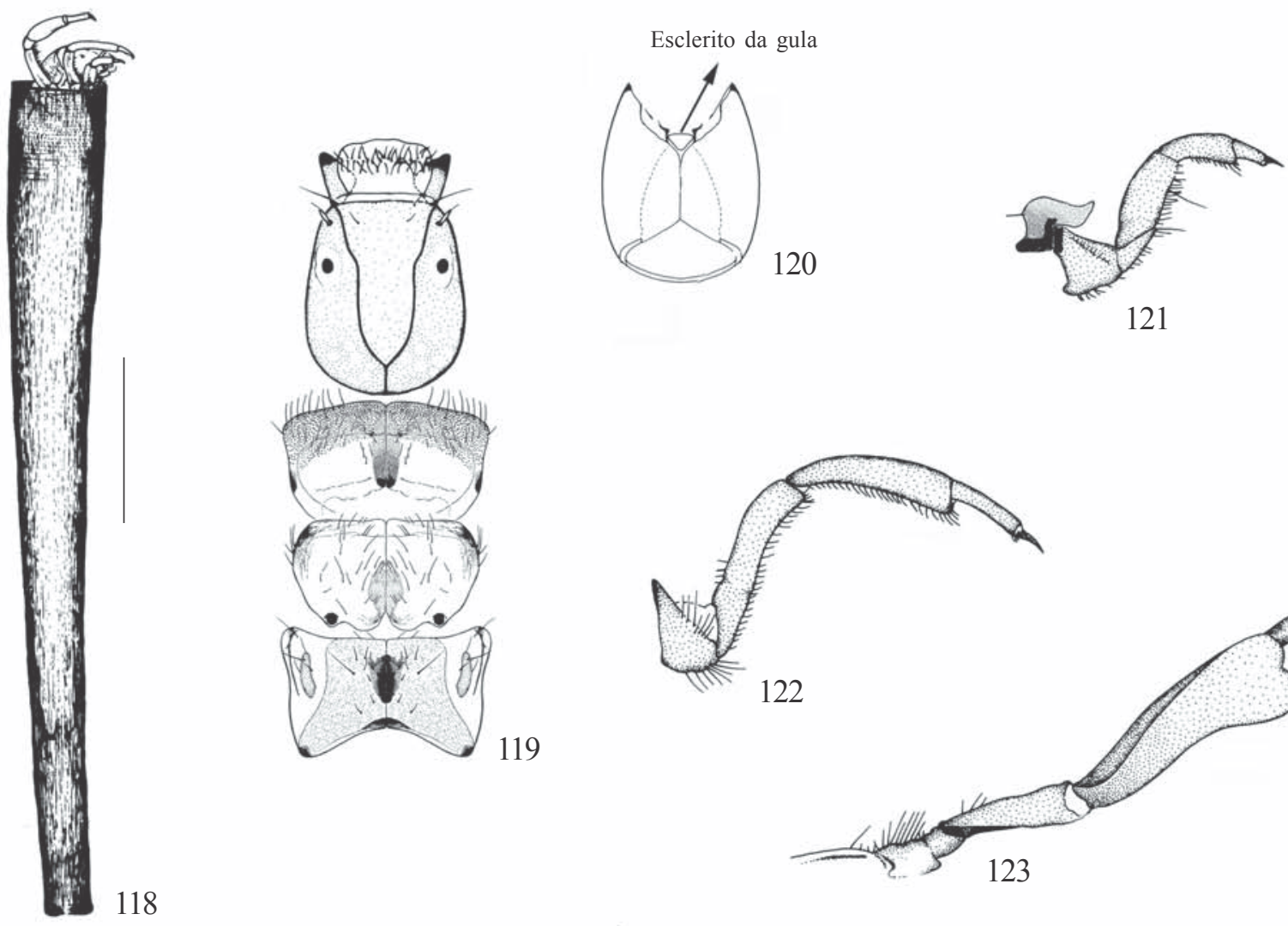

121

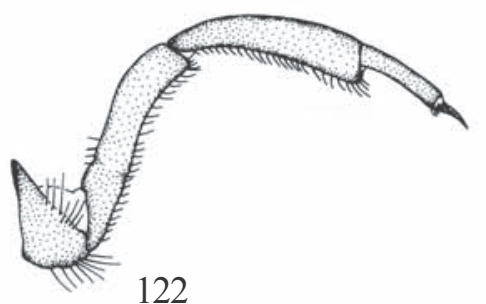

119

122

118
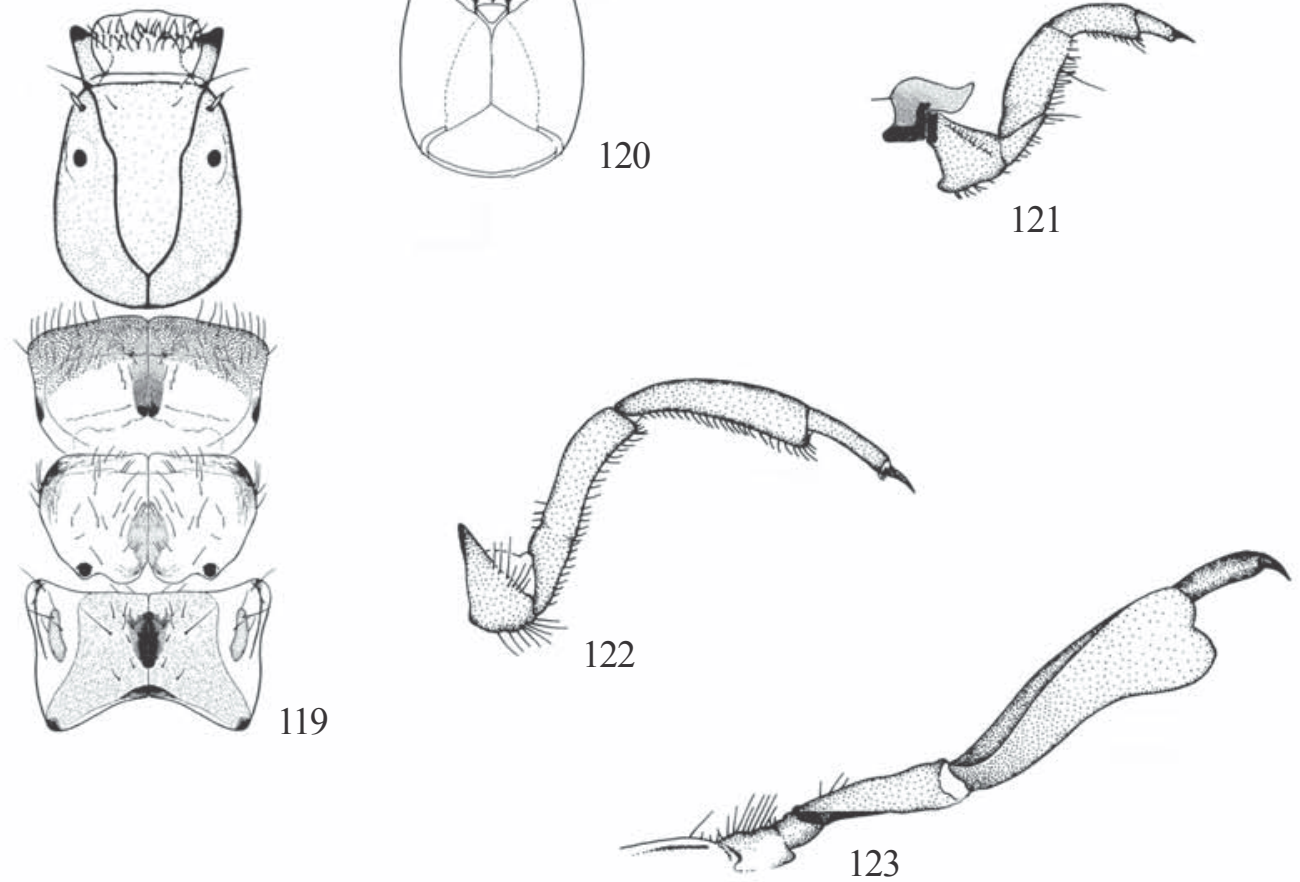

Figs. 114-123. Leptoceridae. 114-117, Triplectides egleri Sattler. 114, larva vista lateral; 115, cabeça e tórax vista dorsal; 116, cabeça vista ventral; 117, abrigo. Escala $=2 \mathrm{~mm} .118-123$. Amazonatolica hamadae Holzenthal \& Pes. 118, abrigo e larva vista lateral; 119, cabeça e tórax vista dorsal; 120, cabeça vista ventral; 121-123, primeiro, segundo e terceiro pares de pernas. Escala $=1 \mathrm{~mm}$. 

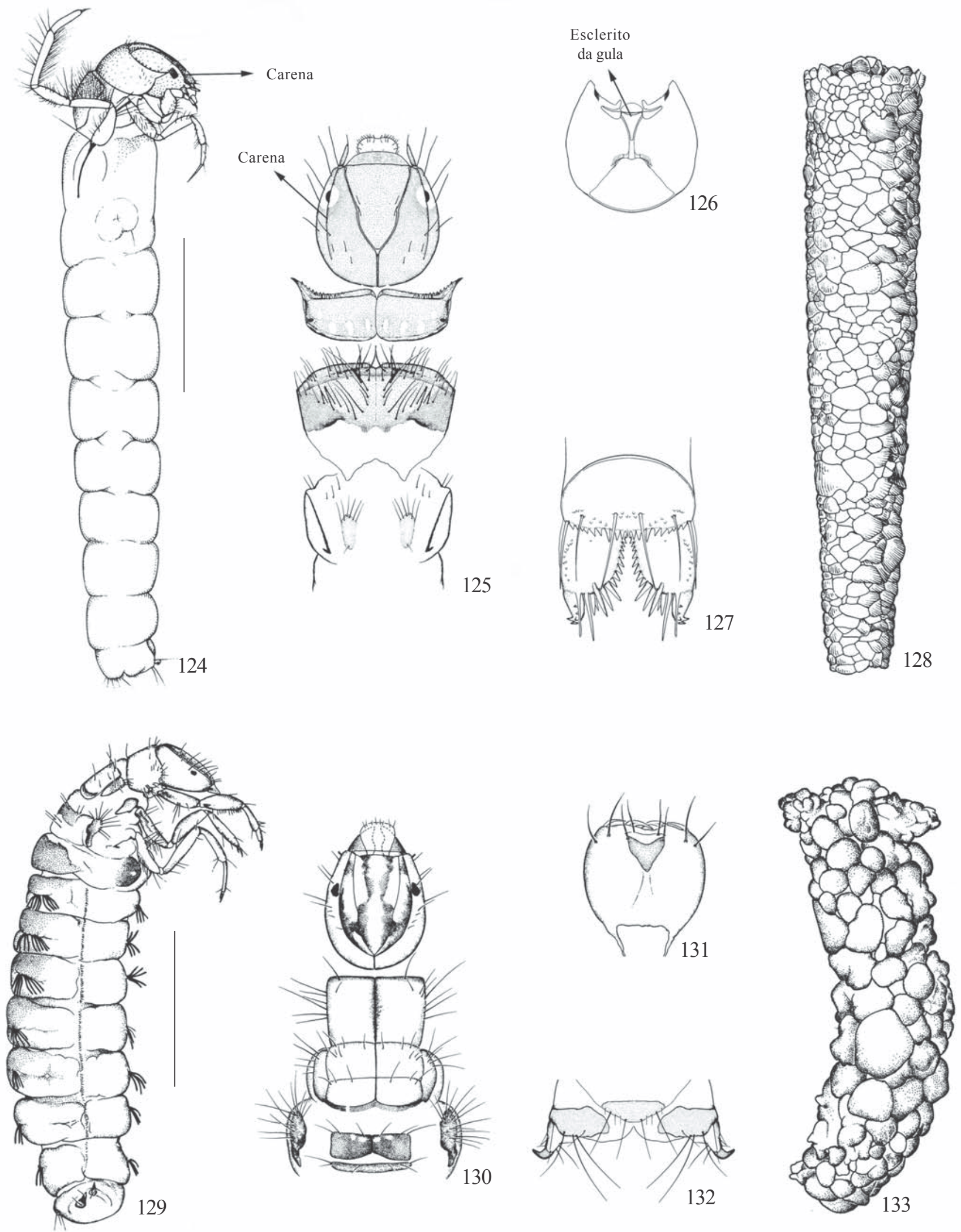

Figs. 124-133. 124-128. Leptoceridae. gênero A. 124, larva vista lateral; 125, cabeça e tórax vista dorsal; 126, cabeça vista ventral; 127, segmento abdominal IX e falsas pernas anais vista dorsal; 128, abrigo. Escala $=1 \mathrm{~mm} .129-133$. Odontoceridae. Marilia sp. 2.129 , larva, vista lateral; 130, cabeça e tórax, vista dorsal; 131, labro; 132, segmento abdominal IX e falsas pernas anais vista dorsal; 133, abrigo. Escala $=2$ mm. 

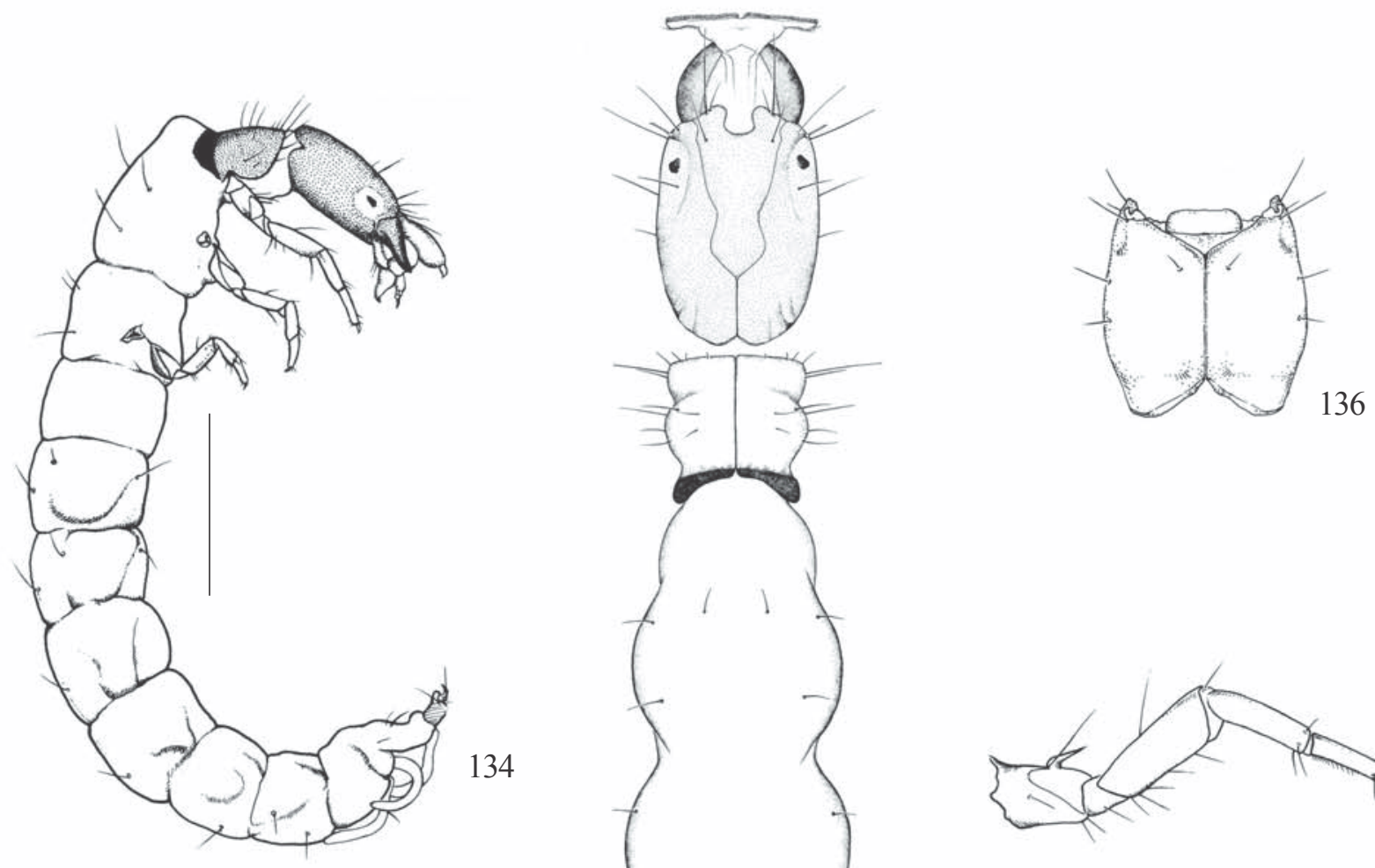

134
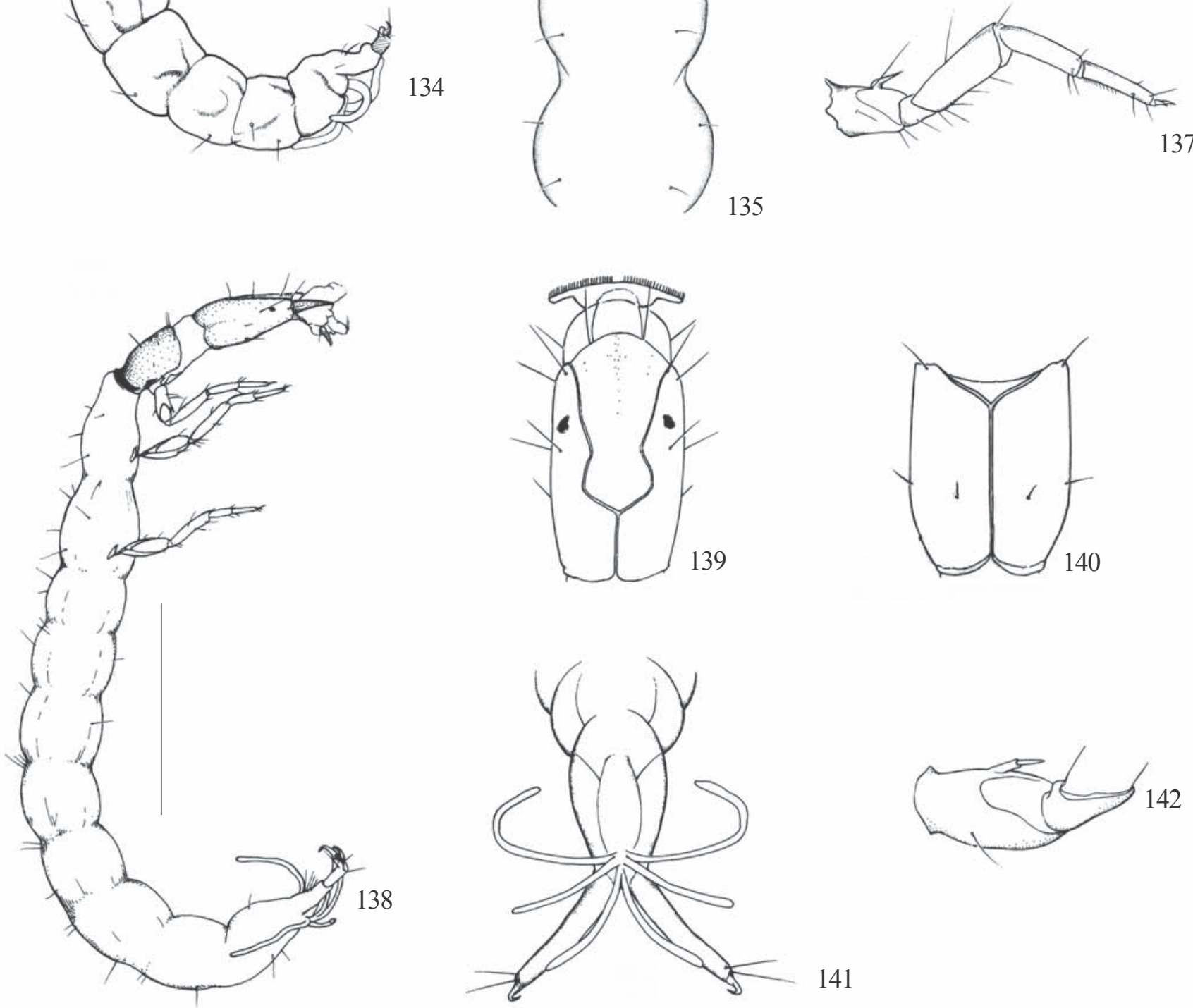

Figs. 134-142. Philopotamidae. 134-137, Chimarra sp. 134, larva vista lateral; 135, cabeça e tórax vista dorsal; 136, cabeça vista ventral; 137, perna anterior. Escala $=2 \mathrm{~mm}$. 138-142. Wormaldia sp. 138, larva vista lateral; 139, cabeça vista dorsal; 140, cabeça vista ventral; 141, segmento abdominal IX e falsas pernas anais vista dorsal; 142, coxa da perna anterior. Escala $=1 \mathrm{~mm}$. 

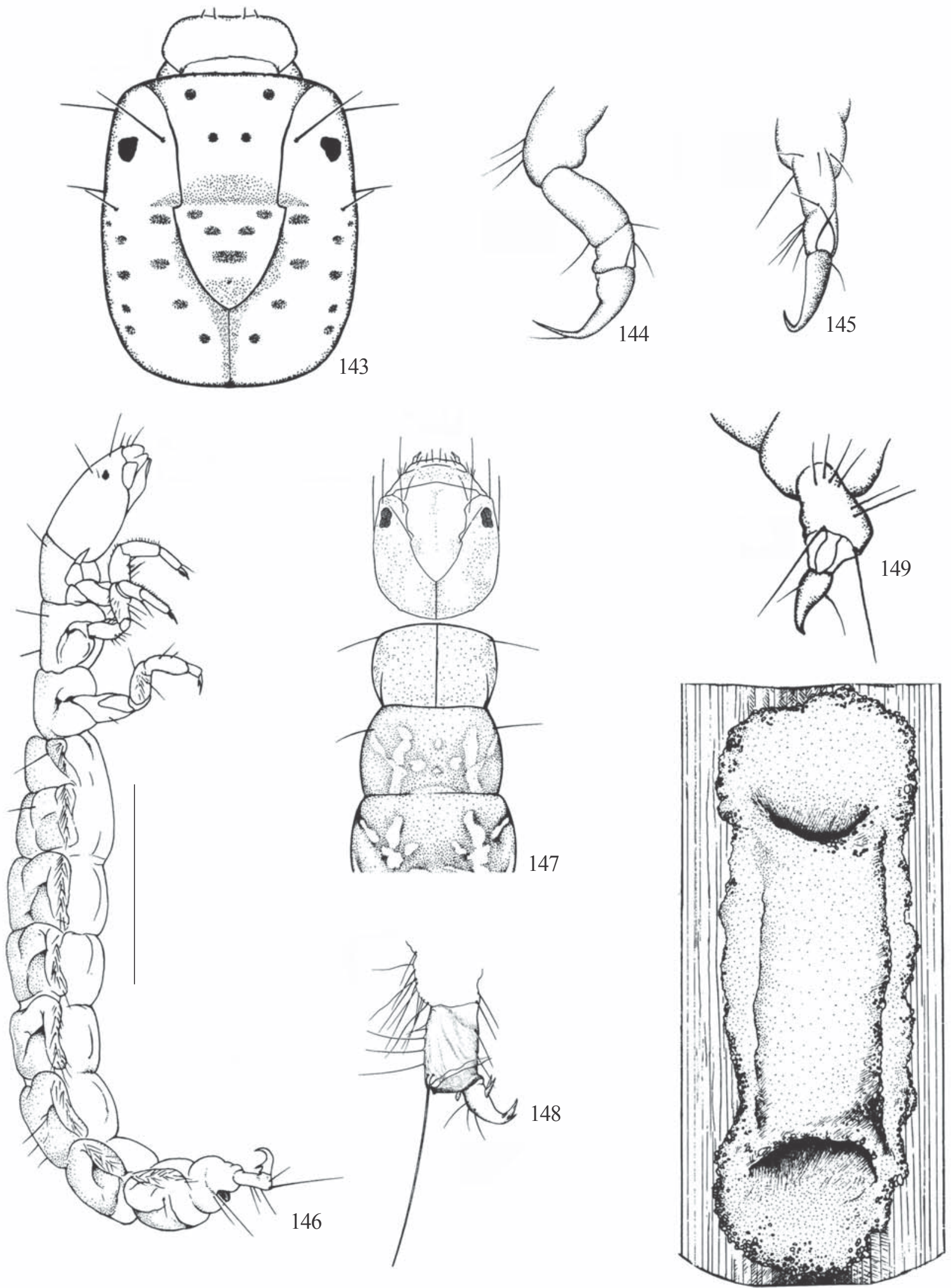

150

Figs. 143-150. Polycentropodidae. 143-145, Cernotina sp. 143, cabeça vista dorsal; 144, falsa perna anal vista lateral; 145 , falsa perna anal vista dorsal. Escala $=1 \mathrm{~mm} .146-150$. Cyrnellus sp. 146, larva vista lateral; 147, cabeça e tórax vista dorsal; 148, falsa perna anal vista lateral; 149, falsa perna anal vista dorsal; 150 , abrigo. Escala $=2 \mathrm{~mm}$. 

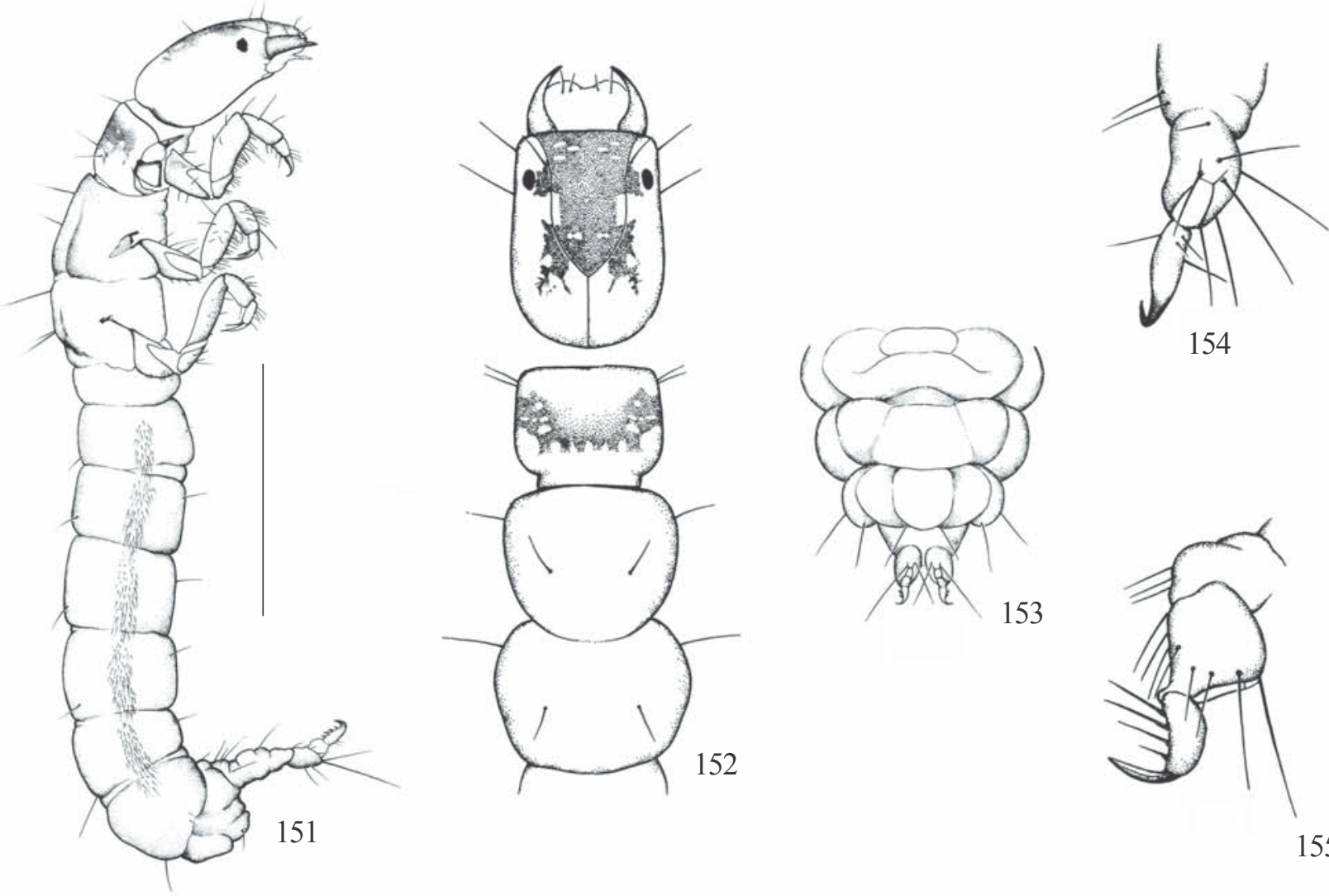

154
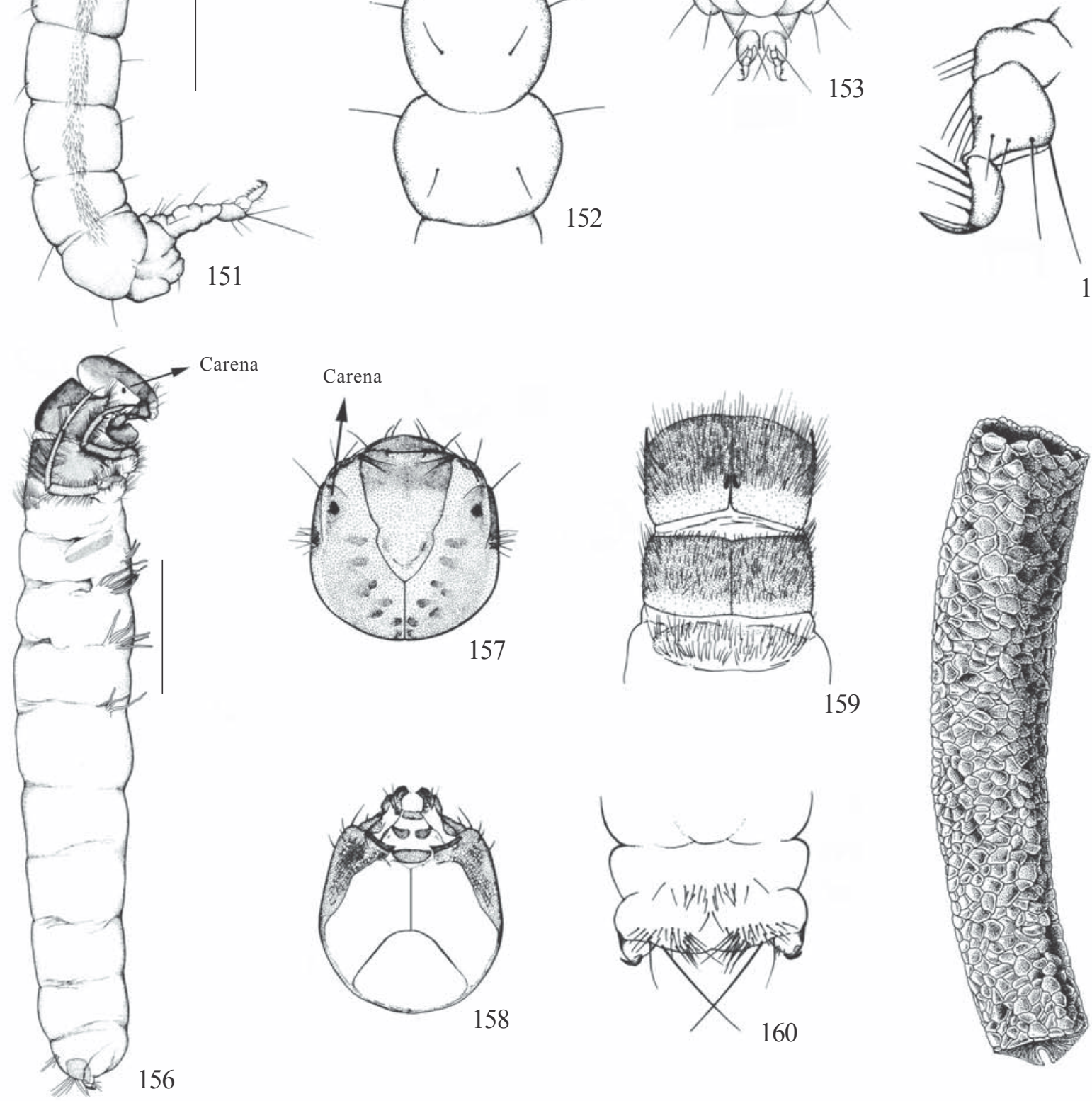

155
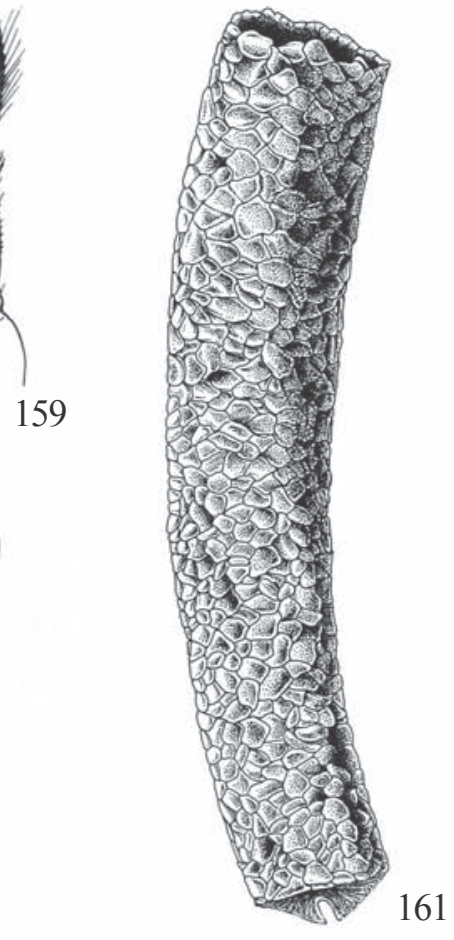

Figs. 151-161. 151-155. Polycentropodidae. Polyplectropus sp. 1. 151, larva vista lateral; 152, cabeça e tórax vista dorsal; 153, segmentos abdominais VII-IX e falsas pernas anais vista dorsal; 154, falsa perna anal vista dorsal; 155, falsa perna anal vista lateral. 156-161. Sericostomatidae. Gênero A. 156, larva vista lateral; 157, cabeça vista dorsal; 158, cabeça vista ventral; 159, tórax vista dorsal; 160, segmentos abdominais VIII e IX e falsas pernas anais vista dorsal; 161, abrigo. Escala $=2 \mathrm{~mm}$. 


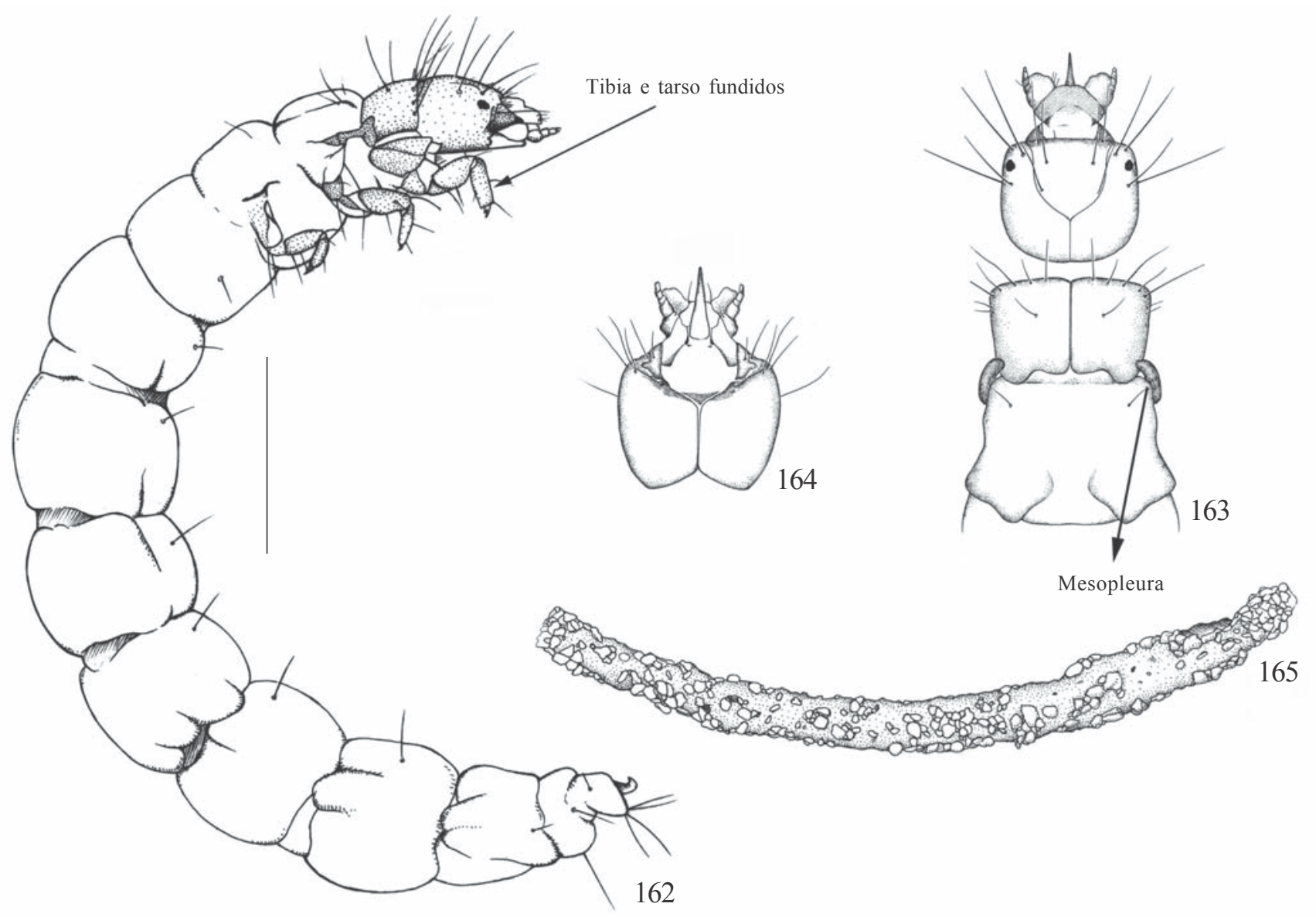

Figs. 162-165. Xiphocentronidae. Machairocentron sp. 162, larva vista lateral; 163, cabeça e tórax vista dorsal; 164, cabeça vista ventral;165, abrigo. Escala $=2 \mathrm{~mm}$.

Recebido em 21.VII.2004; aceito em 10.II.2005 